\title{
Parietal bone mobility in the anesthetized cat
}

\author{
THOMAS ADAMS, PhD \\ RICHARD S. HEISEY, ScD \\ MAHLON C. SMITH, PhD \\ BARBARA J. BRINER, DO
}

To quantify parietal bone motion in reference to the medial sagittal suture, a newly developed instrument was attached to the surgically exposed skull of anesthetized adult cats. The instrument differentiated between lateral and rotational parietal bone movements around the fulcrum of the suture. Bone movement was produced by external forces applied to the skull and by changes in intracranial pressure associated with induced hypercapnia, intravenous injections of norepinephrine, and controlled injections of artificial cerebrospinal fluid into the lateral cerebral ventricle. Responses varied considerably among test animals. Generally, lateral head compression caused sagittal suture closure, small inward rotation of the parietal bones, increased intraventriclar pressure, transient apnea, and unstable systemic arterial blood pressure. Graded increases in intracranial volume produced stepped increases in pressure, lateral expansion at the sagittal suture,

\footnotetext{
From the Departments of Physiology (Drs Adams and Heisey), and Mechanical Engineering (Dr Smith), Michigan State University, and the Department of Biomechanics, Michigan State University-College of Osteopathic Medicine (Dr Briner), East Lansing, Mich.

This research paper was supported by AOA grant No. 89-05-294 and 90-05-294 and by BRSG funds from the College of Osteopathic Medicine, Michigan State University.

Reprint requests to Thomas Adams, $\mathrm{PhD}$, Department of Physiology, Michigan State University, East Lansing, MI 48824-1101.
}

and outward rotation of the parietal bones. We attribute variations in animal response largely to differences in intracranial and suture compliance among them. Cranial suture compliance may be an important factor in defining total cranial compliance.

(Key words: Cranial motion, cranial compliance, cranial suture compliance, intracranial pressure, cerebrospinal fluid, cranial bone mobility)

The cranium has many anatomically distinct bony plates that are complexly articulated along the fractally structured suture lines. ${ }^{1}$ The intricacy of these interdigitated junctions and their apparent solidity, especially in the adult and in dried skulls, has led to the view by many traditionally trained physiologists and physicians that the cranium is a rigid enclosure. ${ }^{2-4}$

For many years, however, some have contended that the skull's bones are mobile at their sutures; also that they bend at these fulcrums in harmony with the complex patterns of intracranial forces dynamically applied by systemic arterial and venous pressures, respiration and cerebrospinal fluid pressure among other phenomena, some of which are yet to be identified. It has been proposed that cranial bones hinge at viscoelastic suture lines to the extent that their motion can be palpated. ${ }^{5}$ Detailed anatomic studies showed that these cranial sutures were innervated and vascularized 
and had both fibrous and elastic connective tissue, but they were not rigidly fused. ${ }^{6}$

Many reports of clinical impressions over nearly the past century have led some groups to believe that there is a wide range of amplitude and time variations in how cranial bones move spontaneously, ${ }^{5,7}$ and how their positions affect developmental and behavioral phenomena. ${ }^{8}$ It is generally accepted, however, that these movements are subtle and can be perceived only with training and practice. It is also conceded that their direct measurement is at best difficult. There is a clear conviction in many circles, nonetheless, that cranial motions provide important diagnostic information $^{5}$ and that affecting them yields therapeutic advantages. ${ }^{8}$

In large part because of the paucity of direct quantitative data about cranial bone motion, the traditional view that the bones of the head are immobile and that the cranial vault is a rigid container has prevailed. In this presumably minimally compliant enclosure, brain tissue, blood, interstitial, and cerebrospinal fluid (CSF) volumes continuously compete for space, as stated in the often cited Monro-Kellie hypothesis. Also within this container, tissue compliances define the relationships between changes in intracranial volume and pressure..$^{9-11}$

Data presented here provide direct quantitative evidence that the parietal bones in the anesthetized cat move both laterally and rotationally in reference to the medial sagittal suture that separates them on the dorsal surface of the skull. We show that these movements are brought about both by external forces applied to the head and by internal ones associated with the peripheral pulse and respiratory movements. We also demonstrate that they move because of changes in intracranial pressure produced by induced hypercapnia and by controlled injections of artificial CSF into a lateral cerebral ventricle. These data support the view that the cranial bones (as represented by the bilateral parietal bones) are not as rigidly articulated as is traditionally supposed. Their motion is large enough in some animals that the compliance of their sutures needs to be considered as a factor in defining total cranial compliance.

\section{Methods}

\section{Measurement of cranial bone movement}

Bidirectional cranial bone motion relative to the skull's sagittal suture was measured in our experiments with the isotonic measuring device shown in Figure 1. The instrument was constructed so that on the assumption of symmetry at the sagittal suture, both the rotational movements of the bilateral parietal bones and their relative lateral separation were simultaneously quantified in reference to the midline suture that divides them. Representative calibration data for the instrument obtained by means of a digital micrometer with a resolution of $1 \mu \mathrm{m}$ are shown in Figure 2.

Our device has two sensors, each of which is made of a pair of microfoil strain gages. As shown in Figure 1, one of these sensors is oriented horizontally and measures only the rotation of parietal bones at the sagittal suture. The other is positioned vertically. The two microfoil strain gages (sg) are bonded to opposite sides of two thin, highly compliant steel strips (ss), one of which is vertical and the other horizontal to the dorsal skull surface. Electrical connections link each microfoil gage to a calibrated voltage divider whose output is registered on a calibrated strip chart recorder.

The proximal end of each steel strip (Figure 1) is connected to a common rigid bar (rb at left in the drawing), which is fixed securely to the skull by a stud threaded into one parietal bone. A similarly rigid bar (rb at right in the drawing) is secured to the opposite parietal bone. An adjustable screw at its end touches the distal end of the vertically positioned steel strip during measurement. The distal end of the horizontally positioned steel strip spans the midline sagittal suture (ssut) and holds a pin that rests on the skull surface during a measurement. Rotational movements of the bilateral parietal bones around the hinge point of the sagittal suture are sensed by strain gages bonded to the horizontally mounted strip. Lateral movements of the parietal bones are sensed by the gages in the vertically mounted strip. The steel strips and their mounted gages have a resonant frequency in the order of $15 \mathrm{~Hz}$, which is a response many times faster than required to measure accu-

(continued on page 603) 
The usual dose is $240 \mathrm{mg}$ once daily. If adequate response is not obtained, the dose may be titrated up to $360 \mathrm{mg}$ or $480 \mathrm{mg}$ once daily. VERELAN $120 \mathrm{mg}$ is available for patients requiring lower-dose verapamil therapy.

And now...

\section{THE VERELAN PLEDGE}

Following appropriate dose titration, VERELAN will control blood pressure at the 24 th hour after dosing, or your patients will be reimbursed $100 \%$ of their out-of-pocket costs for their most recent VERELAN prescription. See your VERELAN representative for more details.

References: 1. Carr AA, Bottini PB, Prisant LM, et al. Once-daily verapamil in the treatment of mild-to-moderate hypertension: a double-blind placebo-controlled dose-ranging study. J Clin Pharmacol. 1991;31:144 150. 2. Data on file for VERELAN $240 \mathrm{mg}$, Lederle Laboratories, Pearl River, NY.

\section{Brief Summary}

VERELANo

Verapamill HCI
Sustained-Release Pellet-Filled Capsules

For complete Prescribing Information, consult package insert.

CLINICAL PHARMACOLOGY

Food does not affect the extent or rate of the absorption of verapamil from the controlled release Atrioventricular block can occur in patients without preexisting condition defects (see WARNINGS) Acceleration of ventricular rate and/or ventricular fibrillation has been reported in patients with atrial flutter or atrial fibrillation and a coexisting accessory AV pathway following administration of verapamil (see WARNINGS) In patients with hepatic insufficiency, metabolism is delayed and elimination half-life prolonged up to 14 to
16 hours (see PRECAUTIONS), the volume of distribution is increased, and plasma clearance reduced to about $30 \%$ of normal.

\section{CONTRAINDICATIONS}

Severe LV dysfunction (see WARNINGS), hypotension (systolic pressure $<90 \mathrm{mmHg}$ ) or cardiogenic shock, sick sinus syndrome (if no pacemaker is present), second-or third-degree AV block (if no pacemaker is present), atrial flutterfifibrillation with an accessory bypass tract (eg, WPW or LGL syndromes), (see WARNINGS), hypersensitivity to verapamil.

\section{WARNINGS}

Verapamil should be avoided in patients with severe LV dysfunction (eg, ejection fraction $<30 \%$ ) or moderate-to-severe symptoms of cardiac failure and in patients with any degree of ventricular dysfunction if they are receiving a beta blocker. Control milder heart failure with optimum digitalization and/or diuretics before been reported.

Several cases of hepatocellular injury have been demonstrated to be produced by verapamil. Periodic monitoring of liver function in patients on verapamil is prudent. Some patients with paroxysmal and/or chronic atrial flutter/fibrillation and an accessory AV pathway (eg. WPW or LGL. syndromes) have developed an increased antegrade conduction across the accessory pathway bypassing the AV node, producing a very rapid ventricular response or ventricular fibrillation after receiving IV verapamil (or digitalis). Because of this risk, oral verapamil is contraindicated in such patients. AV block may occur (second-or third-degree, $0.8 \%$ ). Development of marked first-degree block or progression to second- or third-degree block requires reduction in dosage or, rarely, discontinuation and institution of appropriate therapy. Sinus bradycardia, seconddegree AV biock, sinus arrest, pulmonary edema and/or severe hypotension
patients with hypertrophic cardiomyopathy who were treated with verapamil.

\section{PRECAUTIONS}

Verapamil should be given cautiously to patients with impaired hepatic function (in severe dystunction use about $30 \%$ of the normal dose) or impaired renal function, and patients should be monitored for abnormal prolongation of the PR interval or other signs of overdosage. Verapamil may decrease neuromuscular trans. mission in patients with Duchenne's muscular dystrophy and may prolong recovery from the neuromuscular blocking agent vecuronium. It may be necessary to decrease verapamil dosage in patients with attenuated neuromuscular transmission. Combined therapy with beta-adrenergic blockers and verapamil may result in additive negative effects on heart rate, atrioventricular conduction and/or cardiac contractility; there have been reports of excessive bradycardia and AV block, including complete heart block. The risks of such com: bined therapy may outweigh the benefits. The combination should be used only with caution and close moni-
toring. Decreased metoprolol clearance may occur with combined use. Chronic verapamil treatment can
VERELAN* verapamil HCI

increase serum digoxin levels by $50 \%$ to $75 \%$ during the first week of therapy, which can result in digitalis toxicity. In patients with hepatic cirrhosis, verapamil may reduce total body clearance and extrarenal clearance of digitoxin. The digoxin dose should be reduced when verapamil is given and the patient carefully monitored. Verapami wil usually have an additive effect in patients receiving blood pressure-loweriti agents. Disopyramide should not be given within 48 hours before or 24 hours after verapamil administration. duction, and repolarization. Combined verapamil and quinidine therapy in patients with hypertrophic cardiomyopathy should be avoided, since significant hypotension may result. Verapamil has been given concomitantly with short-and long-acting nitrates without any undesirable drug interactions. Interaction be Ween cimetidine and chronically administered verapamil has not been studied. In healthy volunteers, clearlowering of serum lithium levels or increased sensitivity to lithium. Patients receiving both drugs must be monitored carefully.

Verapamil may increase carbamazepine concentrations during combined use. Rifampin may reduce vera-

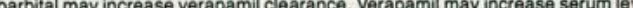
els of cyclosporine. Concomitant use of inhalation anesthetics and calcium antagonists needs careful titration to avoid excessive cardiovascular depression. Verapamil may potentiate the activity of neuromuscu. lar blocking agents (curare-like and depolarizing); dosage reduction may be required. Adequate animal carcinogenicity studies have not been pertormed. One study in rats did not suggest a tumorigenic potential, an verapamil was not mutagenic in the Ames test. Pregnancy Category C: There are no adequate and well. controlled studies in pregnantwomen. This drug should be used during pregnancy, labor, and delvery only clearly needed. Verapamil is excreted in breast milk; therelore, nursing should be discontinued during vera. ADVERSE REACTIONS

Reversible (upon discontinuation of verapamil) nonobstructive, paralytic ileus has been infrequently reported in association with the use of verapamil.

In clinical trials with 285 hypertensive patients on VERELAN for more than 1 week, the following adverse reactions were reported: constipation $(7.4 \%)$; headache $(5.3 \%)$; dizziness $(4.2 \%)$; lethargy $(3.2 \%)$; dyspepsia $(2.5 \%)$; rash $(1.4 \%)$; ankle edema (1.4\%); sleep disturbance $(1.4 \%)$; myalgia (1.1\%). In clinical trials of other formulations of verapamil $\mathrm{HCl}(\mathrm{N}=4,954)$, the following reactions have occurred at rates greater than (2.2\%) constipation $(7.3 \%)$; dizziness $(3.3 \%)$; nausea $(2.7 \%)$; hypotension $(2.5 \%)$; $\theta$ dema $(1.9 \%)$; block-total $1^{\circ}, 2^{\circ}, 3^{\circ}(1.2 \%) ; 2^{\circ}$ and $3^{\circ}(0.8 \%)$; flushing $(0.6 \%)$; elevated liver enzymes (see WARNINGS). The following reactions, reported in $1.0 \%$ or less of patients, occurred under conditions (open trials, marketing experience) where a causal relationship is uncertain. Cardlovascular: angina pectoris, atrioventricular dissociation, chest pain, claudication, myocardial infarction, palpitations, purpura (vasculitis), syncope. Digestive System: diarrhea, dry mouth, gastrointestinal distress, gingival hyperplasia. Hemic and Lym ders, insomn muscle cramps, paresthesia psychotic symptoms, shakiness, somnolence. Respiratory: dyspnea Skin: arthralgia and rash, exanthema hair loss, hyperkeratosis, maculae sweating urticaria dyspnea. Skin: arthralgia and rash, exanthema, hair loss, hyperkeratosis, maculae, Sweating, urticaria, mastia, impotence, increased urination, spotty menstruation.

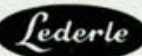
Manufactured for
LEDERLE LABORATORIES DIVISION American Cyanamid Company
Pearl River, NY 10965

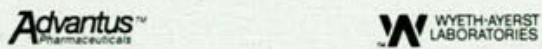




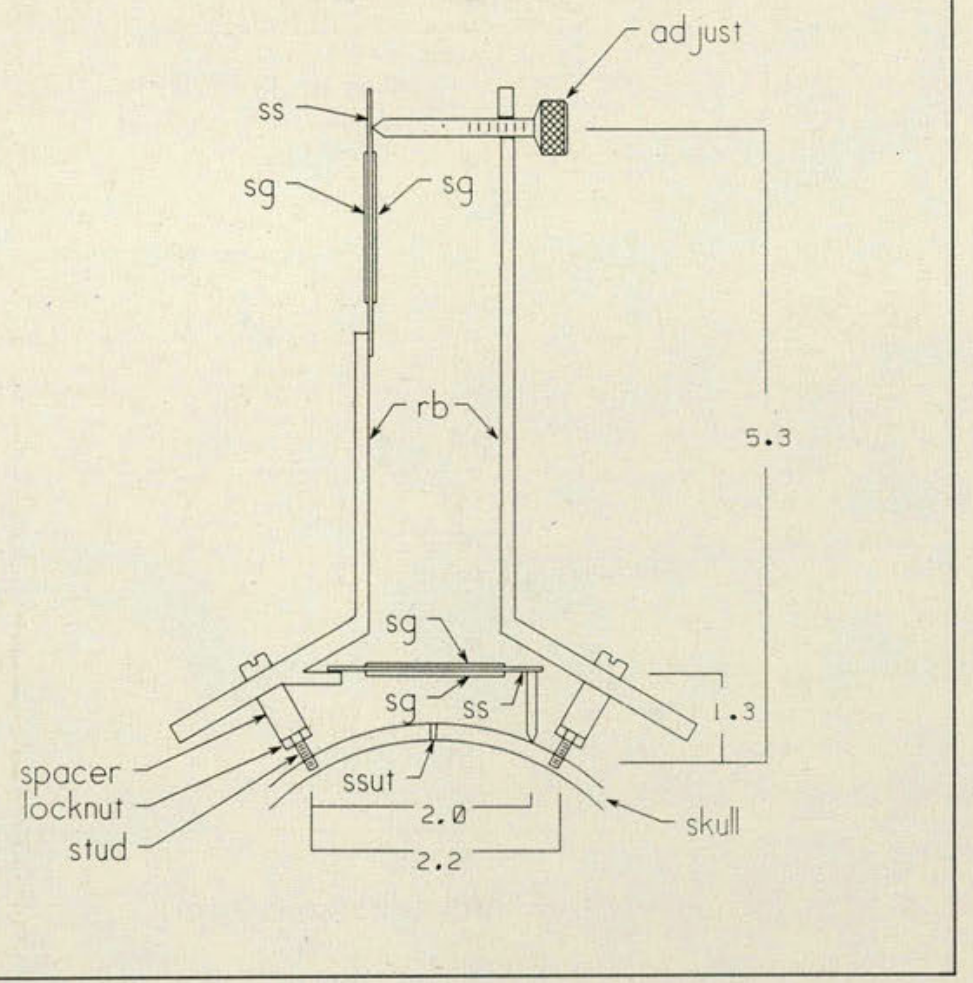

Figure 1. Device used to measure two-dimensional movements of cranial bones in anesthetized animals. Dimensions are in centimeters. SS indicates steel strips; $\mathrm{sg}$, strain gages; rb, rigid bar; ssut, sagittal suture.

rately the slow movements of the cranial bones.

There are different ways the vertically positioned strain gage (Figure 1) could be activated. It could be displaced just by a change in the lateral separation of the parietal bones at the sagittal suture. In this case, narrowing of the suture would bring the bilateral sections of the device closer together and cause a change in voltage output of the calibrated amplifier (Figure 2) to which the bonded pair of strain gages are connected. Also in this case, widening of the suture would separate the left and right supports, causing a voltage output in the opposite direction of the preloaded sensor.

The vertical gage, however, could also be deformed were there no change in suture width, but instead just rotation of the parietal bones around the fulcrum of the sagittal suture. A net outward (counterclockwise) rotation would lever the vertical elements of the device to be closer. A net inward (clockwise) rotation would separate them. Calibrated voltage outputs of both sensors, of course, would be appropriately triggered and recorded. Our measuring device could be affected in a third way if both lateral and rotational movements of the parietal bones were to occur simultaneously in any combination of directions. We use a series of equations based on the geometry of the animal's head and the dimensions of the measuring device (Figure 1) to distinguish lateral from rotational movements of the parietal bones when they occur simultaneously, as described in the Appendix.

There are two stages in the analysis we make to distinguish lateral from rotational parietal bone movements. The first is to have the horizontally and vertically positioned gages (Figure 1) independently calibrated (Figure 2) and to record separately the output voltage of each as a function of time on different but adjacent channels of a calibrated strip chart re- 


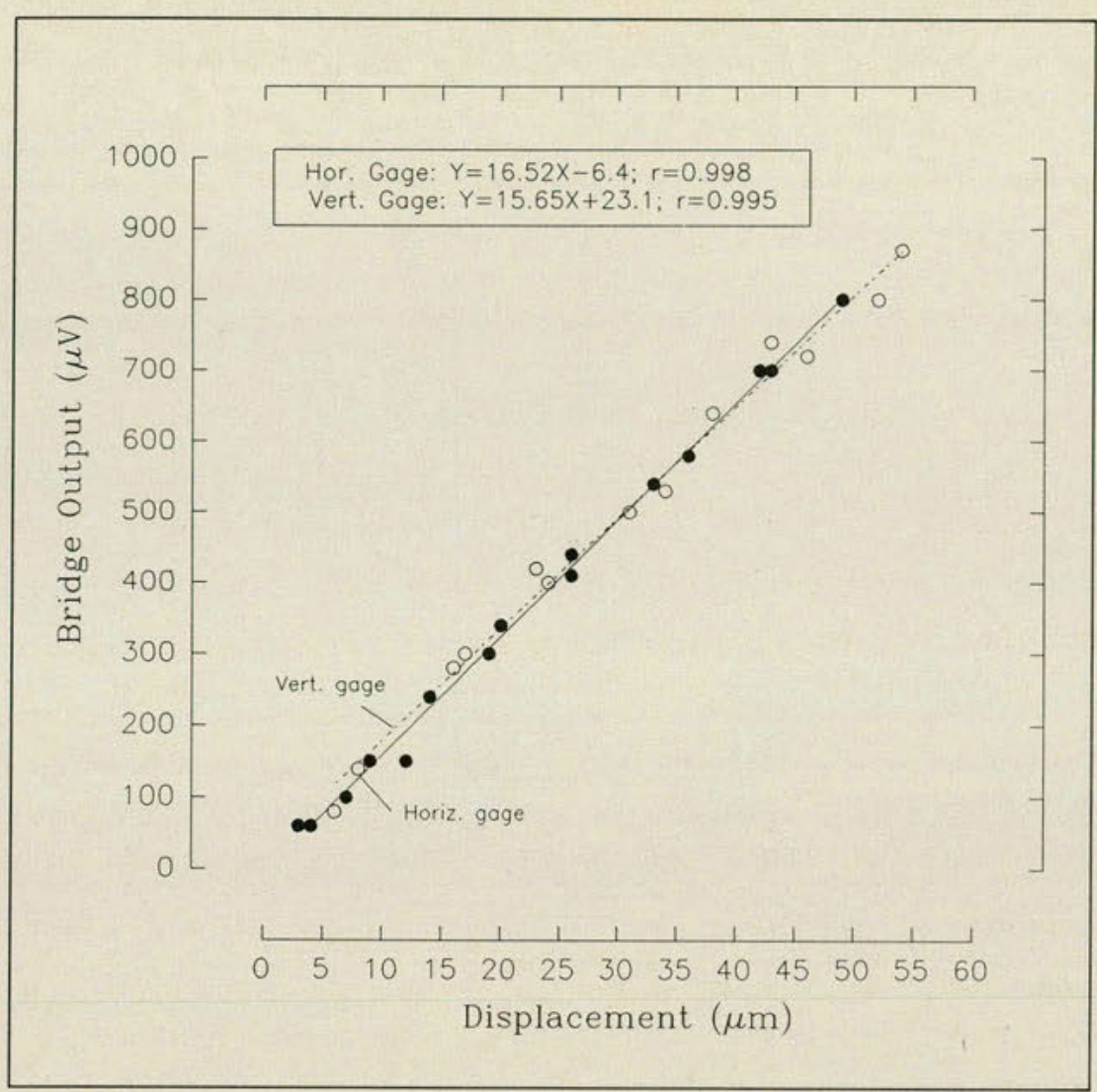

Figure 2. Representative independent calibrations for horizontally mounted and vertically mounted sets of strain gages used to measure parietal bone movements (Figure 1). In each graph, bridge amplifier voltage outputs are shown as functions of digital micrometer-controlled displacements of each sensor. The equation with each graph shows the best-fit linear regression relationship for the multipoint calibration and its correlation coefficient. Multiple calibrations show that the device is free of hysteresis and retains its deformationvoltage output relationships for many months.

corder. These data are called measured movements (MMV). Many data about parietal bone movements are documented in this way in the illustrations in this report. The second stage in our analyses is to distinguish quantitatively lateral movement of the parietal bones from their rotation (see Appendix). Data calculated in this way are called computed movements (CMV). In the simplest case, were no net rotational movements recorded, then all recorded changes from the vertically positioned gage are assumed to directly reflect lateral movement at the sagittal suture (that is, MMV = CMV).

Illustrations showing data for lateral and rotational bone movement are reproductions of directly recorded voltages from our vertical and horizontal strain gages shown with a cor- responding calibration. They report MMVs. Calculated bone movement for which the effects of bone rotation are considered are reported in legends and in the text. They report CMVs and angles (in degrees) of bone rotation. When data about bone rotation were unavailable, our only estimate of lateral motion is the corresponding MMV.

At the beginning of each experiment, the head of the anesthetized cat was rigidly fixed in a stereotaxic frame. This kind of restraint provided not only a three-dimensional reference for inserting a cannula into the brain's lateral ventricle, but also provided stability for attaching the cranial measuring device (Figure 1). A midline skin incision from the level of the supraorbital ridges to the back of the 
skull exposed muscle and connective tissue that were dissected free, excised, or retracted. The dorsal skull surface was cleaned, and threaded studs to which the measuring device is attached were threaded through the full depth of the skull. One 4-40 screw rounded at its end was secured in each parietal bone approximately $1 \mathrm{~cm}$ posterior and lateral to the bregma. Fast-setting dental acrylic was applied to each threaded stud at the surface of the skull to assure its immobility. Exposed tissue and bone were sprayed with medical-grade silicone to minimize their drying. The animal's head and neck were draped loosely during a test.

\section{Measurement of physiologic parameters}

Arterial blood pressure and heart rate were recorded with a cannula inserted into the femoral artery. The cannula was connected to a pressure transducer whose voltage output was recorded on a Grass strip chart recorder. A cannula inserted in the femoral vein was used for injection of drugs and for supplementing anesthesia. Lateral and rotational cranial bone movements were recorded on adjacent channels of the recorder. Respiratory rate and depth were recorded in a similar way with the use of a pneumotachograph attached to the outflow orifice of a low deadspace respiratory valve leading from an endotracheal tube arranged so that the animal could breathe spontaneously or be artificially ventilated. The animal's body temperature was held near-constant at $38^{\circ} \mathrm{C}$ (rectal) by having it rest on a controlled heating pad. Body temperature was monitored continuously.

Intracranial pressure was recorded with a 20-gauge needle inserted into a lateral brain ventricle through a $2-\mathrm{mm}$ diameter hole drilled in the dorsal skull surface. The needle was positioned stereotaxically at the beginning of the experiment (anteroposterior $=$ nominally (nom) $13.5 \mathrm{~mm}$; lateral $=$ nom $2.5 \mathrm{~mm}$; vertical $=$ nom $17.0 \mathrm{~mm}$ ). Dental acrylic was used to seal the hole around the shaft of the needle and to hold it rigid when the animal was removed from the stereotaxic frame. The needle inserted into the lateral ventricle remained in place throughout all tests and served as a site for injecting or removing CSF during an experiment.

All experiments were performed on adult domestic cats that remained fully anesthetized (sodium pentobarbital, $36 \mathrm{mg} / \mathrm{kg}$ intraperitoneally) in all procedures. Animals were killed at the end of the experiment with a lethal bolus injection of anesthetic. Intracranial volume was determined for each animal by decapitating it, chemically removing the soft tissue including the brain from the skull, then filling the skull with small-diameter (size 6; nom $0.2 \mathrm{~mm}$ ) glass beads whose density was known. This information allowed expressing injected volumes of artificial CSF not only in terms of the volume itself, but also as a percent increase in cranial volume which, of course, was different for animals with different cranial volumes.

\section{Results}

Except when noted otherwise, all data and copies of records are presented as representative responses among animals in our tests; not all measurements were made on all animals. Because not all animals responded equally well to all imposed independent variables, data are selectively presented to show least equivocally the phenomena we wish to demonstrate. In no case are data presented from a single test on only one animal. No phenomenon is documented that could not be clearly demonstrated in multiple tests on different animals, even though not all animals responded in the same quantitative way to the same test.

\section{Cranial bone movement: Spontaneous activity}

The upper traces in Figure 3 report the pattern of voltage output from our device (Figure 1) and the amplifier to which it was connected and calibrated while the instrument was activated but not attached to an animal's skull. The indicated stability of these traces was commonly recorded at the beginning of each day of experimentation for all animals. It is the stability of the baseline traces in this record and others similar to it to which all experimental data are compared and analyzed. The lower traces in Figure 3 were recorded with 
our device when it was attached to the head of a spontaneously breathing and anesthetized animal that rested outside of a stereotaxic frame.

Data in the lower half of Figure 3 show the complex wave form for lateral and rotational parietal bone motion we commonly found for our test animals. Even cursory examination shows that the traces have several subcomponents, some of which are shown in later records to be associated with the animal's heart rate and arterial blood pressure, others related to its respiratory patterns. It is patterns of spontaneous activity similar to those shown in this record against which we compare parietal bone movements induced by the internal and external forces applied to the skull that we structured in our experimental protocols.
Baseline
No. 3598

horizontal gage

vertical gage

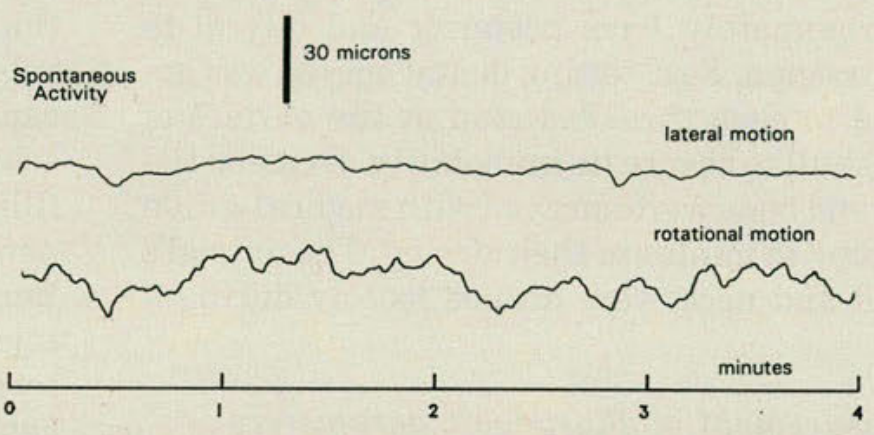

Figure 3. Upper half: Trace of amplifier output for cranial motion detector (Figure 1) when activated but not attached to experimental animal. Lower half: Trace of spontaneous lateral and rotational parietal bone movement across sagittal suture as experimental animal rested without head restraint. Data for spontaneous activity were obtained within minutes after recording those for baseline.

\section{Cranial bone movement imposed by external forces}

Head compression by a manually applied force. The initial phase of our tests was to secure the animal's head in a stereotaxic frame in order to insert and seal the cannula into the lateral cerebral ventricle and to attach our device (Figure 1), which measured parietal bone movement relative to the sagittal suture. The animal's head was then released from the stereotaxic frame and placed to rest without restraints on a padded surface at body level. After baselines for spontaneous bone movement became stabilized, we began our tests, one of which was to use a thumb and forefinger to compress and hold firmly the sides of the resting animal's head. Typically after $1 \mathrm{~min}-$ ute, this pressure was released. Data in Figure 4, top frame show the effects of these maneuvers. Data in Figure 4, bottom frame show the results of a similar experiment when pressure was applied downward on the animal's head at the sagittal suture. Care was taken, of course, not to touch the measuring device itself in any of our tests.
Coincidentally with the application of inward directed force on the sides of the head (Figure 4, top frame), the parietal bones moved laterally closer and underwent inward rotation. Intracranial pressure increased, and there were transient changes in heart rate and in respiratory rate and depth. Release of the manually applied force was accompanied by a return of the cranial bones to their near-rest position, a lowering in CSF pressure, and stabilization of the animal's cardiovascular and respiratory responses. These responses were easily duplicated in subsequent tests on the same animal.

Although responses were similar for some animals when forces were applied externally to the skull, they were very different among others. Those with apparently less compliant sutures showed less parietal bone movement and fewer changes in intracranial pressure and physiologic responses than did those whose heads were more easily deformed. Some animals showed no responses at all to head compression. For those that did, however, their responses were highly reproducible for the same 
animal and generally followed the forms shown in Figure 4, top frame.

Despite the clear effects of increasing intracranial pressure by lateral compression (Figure 4, top frame), downward force applied to the sagittal suture (Figure 4, bottom frame) elicited no cardiovascular or respiratory effects. Both lateral and rotational cranial bone movements were in the opposite direction to those recorded when the head was laterally compressed; respiration rate and depth, heart rate, and blood pressure were comparatively unaffected. Cranial motions presented in Figure 4 , bottom frame are interpreted to indicate a widening of the sagittal suture accompanied by an outward rotation of the parietal bones.

Cranial suture compression and extension by a mechanical device. We used a more controlled method than manual compression of the animal's head to demonstrate cranial bone movement. For these tests, one 4-40 sized, roundheaded screw was attached to each of the parietal bones approximately $0.5 \mathrm{~cm}$ posterior to the attachment site of the instrument we used to measure cranial bone motion (Figure 1). Similarly to the threaded studs that held the cranial motion detector, these screws were firmly threaded through the full thickness of the skull, but with care not to damage the underlying dura, extend into the cranial cavity, or press on brain tissue.

These screws served as the attachment sites for a lightweight, high-compliance spring that applied approximately $2.2 \mathrm{~N}$ force either to compress or extend the parietal bones in reference to the sagittal suture, the choice depending on the orientation of the spring ends to the screws in the parietal bones. In no case was the spring ever attached to, nor did it ever touch, the threaded studs that supported the device measuring cranial motion. Data in Figure 5 show the effects of this controlled compression and extension of the parietal bones in reference to the sagittal suture.

\section{Movement from internal forces}

We used three different techniques to determine how the parietal bones moved in response to intracranial pressure changes. In some animals, we increased such pressure by having the animal breathe $\mathrm{CO}_{2}$-in-air gas mixtures; in others, we injected norepinephrine intravenously; but, in most, we injected controlled volumes of artificial CSF directly into the brain's lateral ventricle.

Effects of induced hypercapnia. Breathing $\mathrm{CO}_{2}$ is widely known to produce an increase in brain blood flow and intracranial pressure, ${ }^{12-14}$ to change cerebral blood volume, ${ }^{15}$ and to alter CSF dynamics. ${ }^{16,17}$ We used induced hypercapnia in our test animals to provide an intracranially directed force to move the parietal bones, as shown in Figure 6, top frame.

The onset of the test animal's spontaneously breathing an $8 \% \mathrm{CO}_{2}$-in-air gas mixture was accompanied by a rise in CSF pressure, an increase in systemic arterial blood pressure, and a corresponding lateral movement of the parietal bones, but no net change in rotational position, at the sagittal suture (Figure 6, top frame). These responses began their return toward pretest levels when the animal again breathed room air. Our impression is that those animals with the more rigid skulls in which parietal bone movement was least affected by external forces are also those in which induced hypercapnia by $\mathrm{CO}_{2}$ breathing had the least effect on suture dimensions.

Effects of injecting norepinephrine and artificial CSF. The bolus intravenous administration of norepinephrine (Figure 6, center frame) transiently elevated intracranial pressure and altered cranial bone alignments.

Mechanical properties of the cranial contents have been of interest in neurosurgical and in neurophysiologic laboratories for many years. ${ }^{18-21} \mathrm{~A}$ common assessment is to calculate cranial compliance, defined as the relationship between a change in intracranial pressure resulting from a corresponding change in intracranial volume. We followed a basic protocol of injecting a bolus of a known volume of artificial CSF into the cerebrospinal fluid system while simultaneously recording intracranial pressure, but also measured parietal bone movement during the procedure.

Figure 6, bottom frame shows responses that are qualitatively representative for all other animals that received volumes of injected ar- 
Figure 4. Top frame: Respiration, systemic arterial pressure, CSF pressure, and cranial motion traces in anesthetized cat at rest without head restraint (before $\mathrm{ON}$ ), after lateral manual compression of the temporal bones, and after their release (OFF). Calculated for time = 1.5 minute, $C M V=220 \mu \mathrm{m}$, and rotation $\delta$ $=0.19$ degree. Bottom frame: Analogous data for animal receiving downward force on sagittal suture when held, then released. Calculated for time $=2.8$ minutes, $C M V=295 \mu \mathrm{m}$ and $\delta=0.28$ degree .
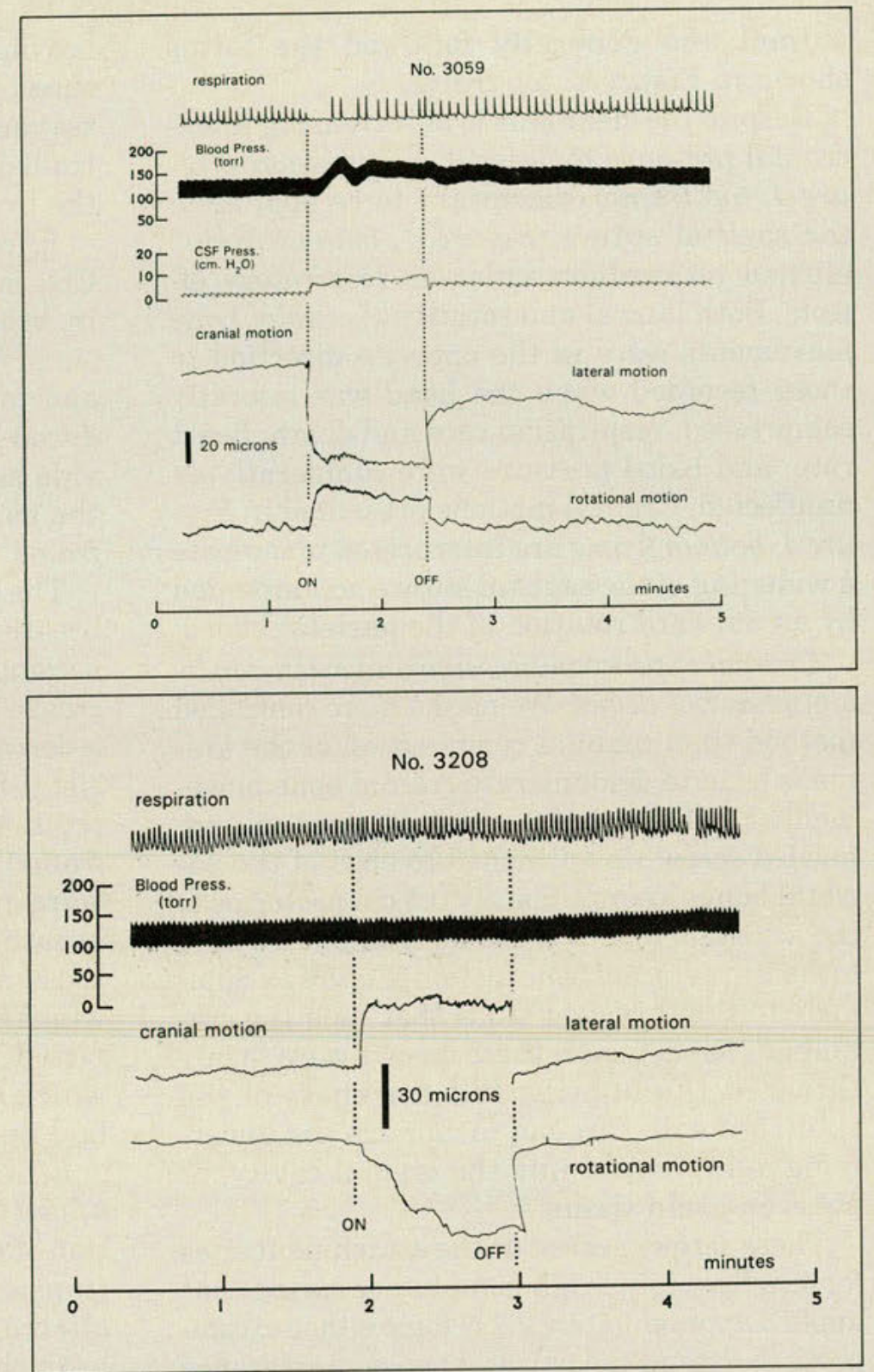

tificial CSF. As soon as the bolus was injected, CSF pressure rose and the parietal bones were displaced. The lateral movement at the sagittal suture was more common than the rotational change in position. These changes in parietal bone alignment matched those produced by pressing on the sagittal suture (Figure 4 , bottom frame). They are interpreted as indicating a widening of the sagittal suture and an outward rotation of the parietal bones. These changes are in a direction one would predict were there an increase in cranial volume and intracranial pressure, as there was when we injected a bolus of artificial CSF (Figure 6 , bottom frame).

Figure 7 shows that parietal bone lateral movement at the sagittal suture progressed precdictably for step-increases in injected volumes of artificial CSF, but its pattern was unique for each animal. Matched tests show that for the same animal, lateral suture movements were virtually the same whether injected volumes were presented either in serial or in random order. Whereas some (animal No. 3059) changed lateral parietal bone position by approximately $70 \mu \mathrm{m}$ for about a $4 \%$ increase in 

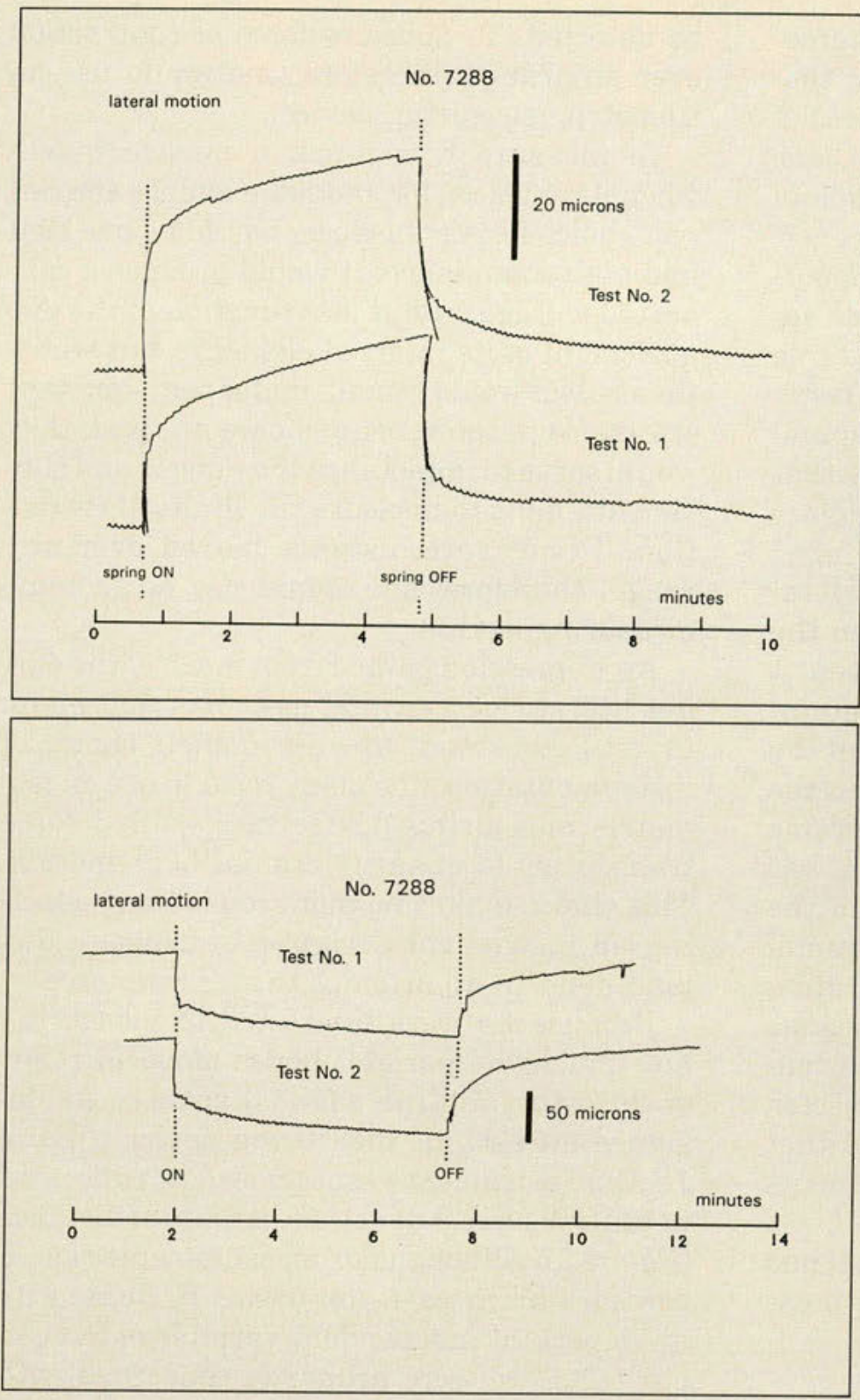

Figure 5. Lateral movements of the parietal bones relative to the sagittal suture when a lightweight spring was first positioned to produce an extending force (top frame, two tests) then a compressing force (bottom frame, two tests) in the same animal. cranial volume, position change for others (animal No. 3615) was no more than about $25 \%$ of this for the same increase in cranial volume. An important point is nonetheless clear. Lateral movement of the parietal bones is idiosyncratically related in a direct way to a controlled increase in intracranial volume (Figure 7), which we assume is causally related to the associated increase in intracranial pressure (Figure 6, bottom frame). How much the parietal bones move either laterally or rotationally for any animal depends, of course, on its intracranial and suture compliances.
Effects of external restraint. How much the parietal bones move in response to a change in intracranial pressure depends not only on the intrinsic mechanical characteristics of the animal's skull, but also on any extracranial restrictions it faces. Data in Figure 8 show one such effect.

A bolus injection of $1 \mathrm{~mL}$ of artificial CSF into an animal's lateral brain ventricle affects the lateral and rotational movements of the parietal bones differently when its head is held rigidly in a stereotaxic frame (Figure 8, top frame) than when it is unrestrained (Figure 
8 , bottom frame). With the earbars of the stereotaxic frame firmly fixed, there was no measurable, net, lateral or rotational movement of the parietal bones (Figure 8, top frame). Clear rotational and lateral transients accompany the increase in intracranial pressure, however, when the head is unrestrained (Figure 8, bottom frame). Also, cardiovascular responses appear more pronounced when the head is free to move; however, the transient effects on respiration appear to be the same. Cerebrospinal fluid pressure transients are similar whether the head was secured or unrestrained (Figure 8 , top frame and bottom frame).

Spontaneous movements of the parietal bones also appeared to be different when the animal's head was restrained than when it rested unrestrained (Figure 9). Both lateral and rotational parietal bone movements at the sagittal suture appear synchronous when the head was confined in the stereotaxic frame (lower traces in Figure 9). This pattern was lost when the animal was released from the frame (upper traces in Figure 9). The animal for which data are shown in this illustration is also the one that showed the greatest change in lateral parietal bone movement when controlled volumes of artificial CSF were injected into its lateral ventricle (Figure 7) and that responded with large cranial bone movements during induced hypercapnia (Figure 6, top frame). The impression is that this animal had the greatest cranial compliance among those we measured.

\section{Discussion}

Success in quantifying physiologic phenomena for factors of either force or motion requires understanding the differences between isometric and isotonic measurements and selecting an appropriate measuring device. To determine how strongly a muscle contracts, for example, the correct choice for a transducer would be one that had low compliance. It would produce a calibrated voltage output as a function of the force applied to it, but its internal elements would move little when it was under stress. If these elements were deformed easily, then any force greater than that minimally required to set them in motion could not be detected. To measure force of contraction over any range, therefore, one would use an isometric measuring device.

To measure how much a muscle moves when it contracts, for another example, the correct choice for a transducer would be one that had high compliance. It would produce a calibrated voltage output as a function of the displacement of its internal elements, but which themselves would remain under near-constant strain. Were the sensing elements rigid, they would serve to immobilize the muscle and subvert attempts to measure the limits of its motion. To measure distance moved over any range, therefore, one would use an isotonic measuring device.

An impression gained from much of the earlier literature on cranial motion is that many investigators were unwise in their choice of instrumentation. Too many tried to use an isometric measuring device (a so-called force transducer) to quantify cranial bone motion. This choice makes no more sense than selecting an instrument designed to measure displacement in an attempt to measure force.

Because we were interested in measuring how much the parietal bones move in reference to the midline sagittal suture, we designed an isotonic measuring device (Figure 1). This instrument's sensors are not calibrated in units of force, but rather in units of distance (Figure 2). Although our measurements of CSF pressure (Figures 4, top frame, 6, 8) identify an important independent variable of force in our tests, we were primarily concerned with quantifying as the dependent variable how much cranial bones move, not the net forces acting on them.

A practical advantage of the instrument we developed is that it has only two major parts, a left and a right element, each of which is easily attached to the skull with a single stud. Another advantage is the linear and matched calibration of its sensors (Figure 2). Using two (rather than just one) microfoil strain gages for each sensor (Figure 1) eliminates thermal drift and increases sensitivity. An additional advantage is that our instrument is attached directly to bone, not to skin where peripheral vasomotor and muscle contractions might af-

(continued on page 615) 


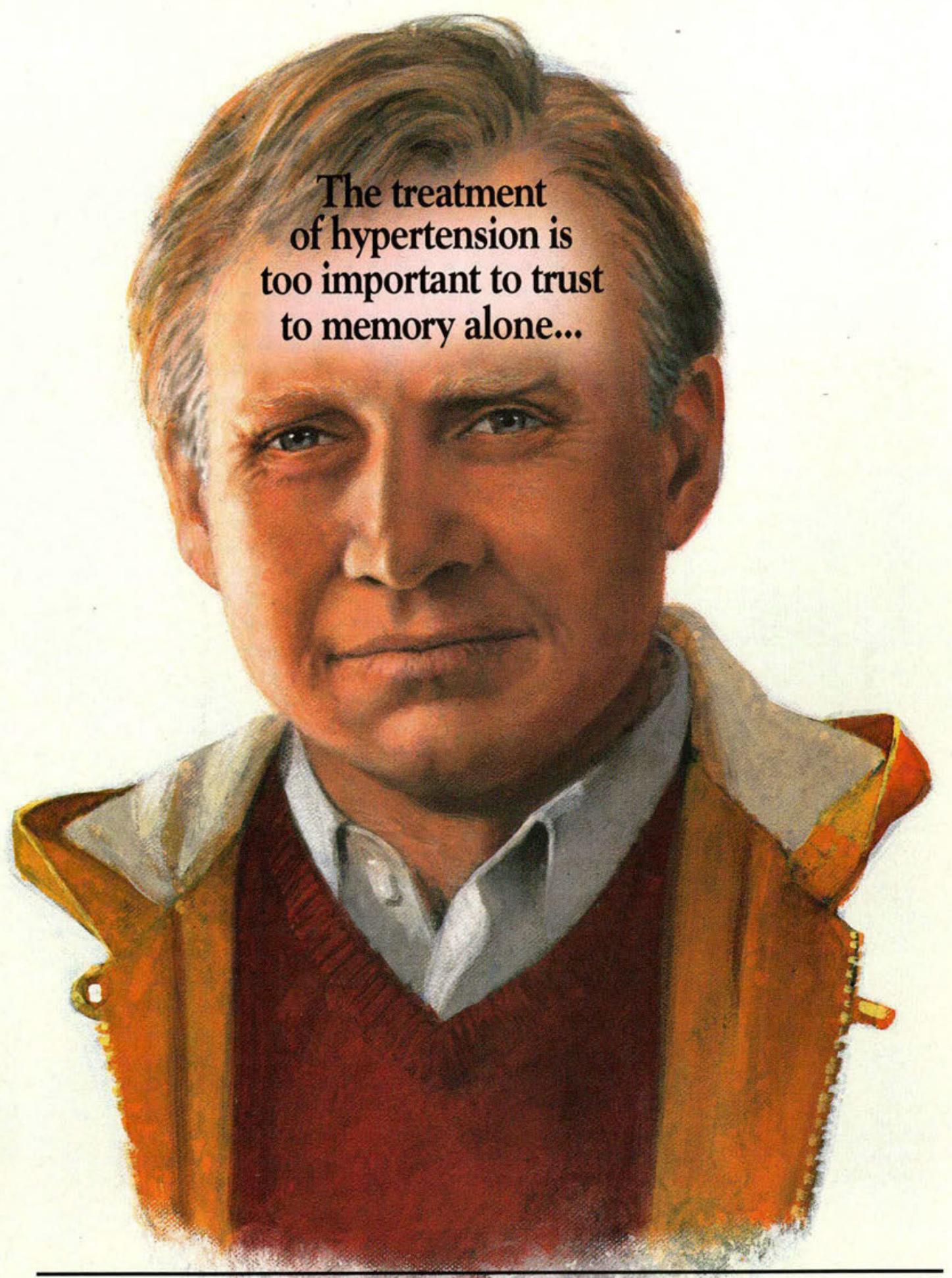

Now...your patients won't forget to take their Clonisp 


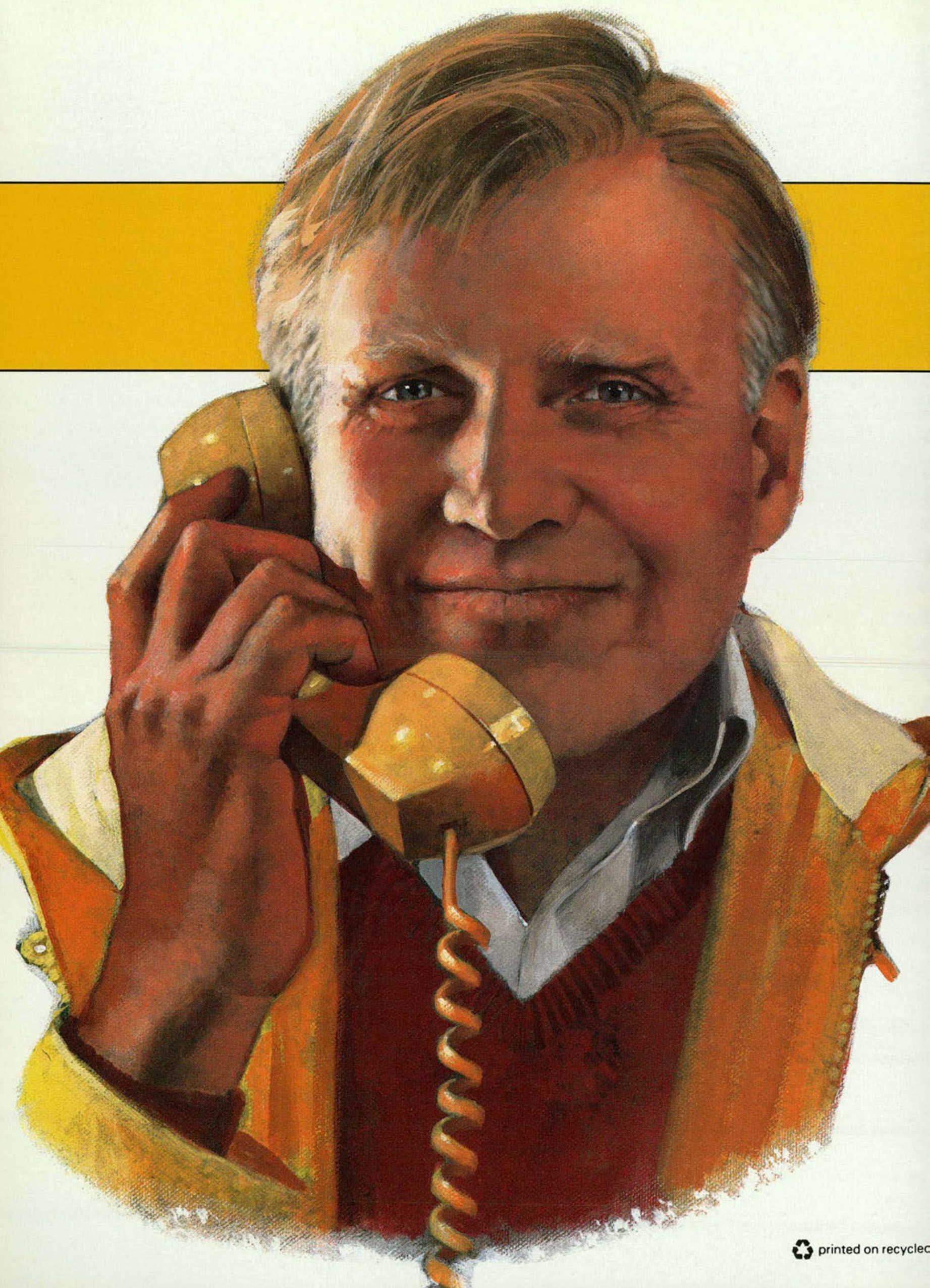




\section{You prescribe the Glanss...}

\section{we'll remind your patients to take it, with a daily* phone call- free.}

The new Calan SR Patients in Compliance ${ }^{\mathrm{TM}}$ program gives you one more excellent reason to start a hypertensive patient on today's most widely used calcium antagonist as monotherapy for mild hypertension': This program should improve the likelihood of effective treatment by helping to eliminate a major cause of noncompliance - forgetfulness.

Now, a Searle representative will personally phone your patients every day* - at times chosen by them - and remind them to take their Calan SR. Patients can even receive their calls in Spanish, if they wish. This valuable service is free but only with your new prescription for Calan SR.

The Calan SR Patients in Compliance program reinforces your instructions, helping patients form a good habit that could be a lifesaver: adhering to your prescribed treatment of a disease that doesn't provide any reminder symptoms of its own.

If you'd like to get your patients started on this important new program, ask your Searle Medical Sales Representative for details. Or phone us, toll-free, at 1-800-2 TAKE RX.

\section{THE CALAN ${ }^{\circ}$ SR PATIENTS IN COMPLIANCE ${ }^{\mathrm{M}}$ PROCRAM}

* Daily phone call for the first week, five calls the second week, three the third, and one the fourth and final week with each new prescription for Calan SR. A reminder phone call will also be made when each refill is due.

$\dagger$ Data on file, Searle. Constipation, which is easily managed in most patients, is the most commonly reported side effect of Calan SR.

Please see brief summary of prescribing information on following page of this advertisement.

(C) 1992 Searle

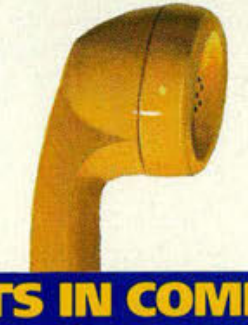




\section{The Calan SR Patients in Compliance ${ }^{\mathrm{TM}}$ program means your patients won't forget their daily dose because we'll call to remind them.}

\author{
Available only with your new \\ Calan SR prescription \\ Helps hypertensive patients \\ get into the habit of taking \\ medication daily
}

$\square$ Enrollment is quick, simple -
and free

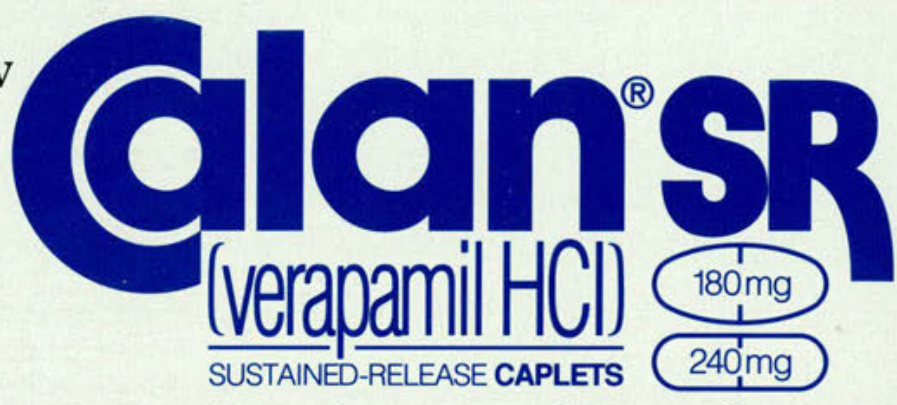

For the many faces of
mild hypertension

\section{BRIEF SUMMARY}

Contraindications: Severe LV dysfunction (see Warnings), hypotension (systolic pressure $<90 \mathrm{~mm} \mathrm{Hg}$ ) or cardiogenic shock, sick sinus syndrome (if no pacemaker is present), 2nd- or 3rd-degree AV block (if no pacemaker is present), atrial flutter/fibrillation with an accessory bypass tract (eg. WPW or LGL syndromes), hypersensitivity to verapamil.

Warnings: Verapamil should be avoided in patients with severe LV dysfunction (eg, ejection fraction $<30 \%$ ) or moderate to severe symptoms of cardiac fallure and in patients with any degree of ventricular dysfunction if they are receiving a beta-blocker. Control milder heart failure with optimum digitalization and/or diuretics before Calan SR is used. Verapamil may occasionally produce hypotension. Elevations of liver enzymes have been reported. Several cases have been demonstrated to be produced by verapamil. Periodic monitoring of liver function in patients on verapamil is prudent. Some patients with paroxysmal and/or chronic atrial flutter/fibrillation and an accessory AV pathway (eg. WPW or LGL syndromes) have developed an increased antegrade conduction across the accessory pathway bypassing the AV node, producing a very rapid ventricular response or ventricular fibrillation after receiving I.V. verapamil (or digitalis). Because of this risk, oral verapamil is contraindicated in such patients. AV block may occur (2nd-and 3rd-degree, $0.8 \%$ ). Development of marked 1st-degree block or progression to 2 nd- or 3rddegree block requires reduction in dosage or, rarely, discontinuation and institution of appropriate therapy. Sinus bradycardia, 2nd-degree AV block, sinus arrest, pulmonary edema and/or severe hypotension were seen in some critically ill patients with hypertrophic cardiomyopathy who were treated with verapamil.

Precautions: Verapamil should be given cautiously to patients with impaired hepatic function (in severe dysfunction use about $30 \%$ of the normal dose) or impaired renal function, and patients should be monitored for abnormal prolongation of the PR interval or other signs of overdosage. Verapamil may decrease neuromuscular transmission in patients with Duchenne's muscular dystrophy and may prolong recovery from the neuromuscular blocking agent vecuronium. It may be necessary to decrease verapamil dosage in patients with attenuated neuromuscular transmission. Combined therapy with beta-adrenergic blockers and verapamil may result in additive negative effects on heart rate, atrioventricular conduction and/or cardiac contractility; there have been reports of excessive bradycardia and AV block, including complete heart block. The risks of such combined therapy may outweigh the benefits. The combination should be used only with caution and close monitoring. Decreased metoprolol and propranolol clearance may occur when either drug is administered concomitantly with verapamil. A variable effect has been seen with combined use of atenolol. Chronic verapamil treatment can increase serum digoxin levels by $50 \%$ to $75 \%$ during the first week of therapy, which can result in digitalis toxicity. In patients with hepatic cirrhosis, verapamil may reduce total body clearance and extrarenal clearance of digitoxin. The digoxin dose should be reduced when verapamil is given, and the patient carefully monitored. Verapamil will usually have an additive effect in patients receiving blood-pressurelowering agents. Disopyramide should not be given within 48 hours before or 24 hours after verapamil administration. Concomitant use of flecainide and verapamil may have additive effects on myocardial contractility. AV conduction, and repolarization. Combined verapamil and quinidine therapy in patients with hypertrophic cardiomyopathy should be avoided, since significant hypotension may result. Concomitant use of lithium and verapamil may result in a lowering of serum lithium levels or increased sensitivity to lithium. Patients receiving both drugs must be monitored carefully. Verapamil may increase carbamazepine concentrations during combined use. Rifampin may reduce verapamil bioavailability. Phenobarbital may increase verapamil clearance. Verapamil may increase serum levels of cyclosporin. Verapamil may inhibit the clearance and increase the plasma levels of theophylline. Concomitant use of inhalation anesthetics and calcium antagonists needs careful titration to avoid excessive cardiovascular depression. Verapamil may potentiate the activity of neuromuscular blocking agents (curare-like and depolarizing); dosage reduction may be required. There was no evidence of a carcinogenic potential of verapamil administered to rats for 2 years. A study in rats did not suggest a tumorigenic potential, and verapamil was not mutagenic in the Ames test. Pregnancy Category $C$. There are no adequate and well-controlled studies in pregnant women. This drug should be used during pregnancy. labor, and delivery only if clearly needed. Verapamil is excreted in breast milk; therefore, nursing should be discontinued during verapamil use.

Adverse Reactions: Constipation $(7.3 \%)$, dizziness $(3.3 \%)$, nausea $(2.7 \%)$, hypotension (2.5\%), headache $(2.2 \%)$, edema $(1.9 \%)$, CHF, pulmonary edema $(1.8 \%)$, fatigue $(1.7 \%)$, dyspnea $(1.4 \%)$, bradycardia: $\operatorname{HR}<50 / \mathrm{min}(1.4 \%)$, AV block: total $1^{\circ}, 2^{\circ}, 3^{\circ}(1.2 \%), 2^{\circ}$ and $3^{\circ}(0.8 \%)$, rash (1.2\%), flushing (0.6\%), elevated liver enzymes, reversible non-obstructive paralytic ileus. The following reactions, reported in $1.0 \%$ or less of patients, occurred under conditions where a causal relationship is uncertain: angina pectoris, atrioventricular dissociation, chest pain, claudication, myocardial infarction, palpitations, purpura (vasculitis), syncope, diarrhea, dry mouth, gastrointestinal distress, gingival hyperplasia, ecchymosis or bruising, cerebrovascular accident, confusion, equilibrium disorders, insomnia, muscle cramps, paresthesia, psychotic symptoms, shakiness, somnolence, arthralgia and rash, exanthema, hair loss, hyperkeratosis, macules, sweating, urticaria, Stevens-Johnson syndrome, erythema multiforme, blurred vision, gynecomastia, galactorrhea/hyperprolactinemia, increased urination, spotty menstruation, impotence.

\section{Ado.ess medicalinquiries to}

$4 / 11 / 91 \cdot P 91 C A 6277 \mathrm{~V}$

\section{Me. Seartio \& Co:}

Information Department

4901 Searle Parkw
Skokie, 1160077

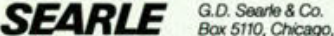

G.D. Searle \& Co.
Bax 5110, Chicago, IL 60680 A92CA6484T 
fect our measurements. Also, it is not hard to make with basic shop tools, and it connects directly to many types of commercially available voltage dividers and recorders commonly found in many laboratories.

A disadvantage of our design is that the vertically positioned gage responds both to lateral and rotational bone movements. Appropriate analyses, however, readily distinguish between these movements (Appendix). A limitation of our experimental design is that our data provide information about mobility of just the sagittal suture. We selected this test site only because of its accessibility, not because it is necessarily representative of any other cranial suture or necessarily the most easily deformed. We make no presumptions about how accurately parietal bone mobility reflects net cranial volume changes.

Data in Figures 4 and 5 clearly answer the central question we have asked of our data: Do the parietal bones move in reference to the sagittal suture? Our data show that compressing forces applied laterally to the head close the midline suture and rotate the parietal bones inward (Figure 4, top frame). Pressing down on the sagittal suture widens it and rotates the parietal bones outward (Figure 4, bottom frame). The CMVs in these tests are in the order of $0.25 \mathrm{~mm}$, but rotation angles are small, although they are convincingly documented as MMVs. Although the changes in intracranial pressure measured in a cerebral ventricle are small during head compression (Figure 4, top frame), the maneuver nonetheless precipitates both respiratory and cardiovascular effects.

Data in Figure 5 show that tissue in the sagittal suture undergoes at least two distinct phases of deformation when a controlled and steady force is applied to the parietal bones, then released from them. The displacement measured as MMVs is a response common to both extending and compressing forces, and common also to both the application and the release of these forces. There is an initial fast change in suture position followed by a slower adjustment during the period of force application and after it is removed. For an extending force (Figure 5, top frame), the initial phase resulted in separation of the parietal bones by approximately MMV $=20 \mu \mathrm{m}$, with the final displacement being about twice that distance.

Compression of the sagittal suture by the spring produced an initial MMV of approximately $50 \mu \mathrm{m}$ (Figure 5, bottom frame), followed by a slower movement in the same direction of about an additional $40 \mu \mathrm{m}$. These data indicate the sagittal suture had a range of motion documented as MMV of about 130 $\mu \mathrm{m}(40 \mu \mathrm{m}$ extension $+90 \mu \mathrm{m}$ compression $)$. Results from similar tests on other animals show this to be comparatively large.

There were considerable differences among animals in how their cranial bones responded to the spring forces we applied to them. Much like the differences we found with manually applied forces to the skull (Figure 4), some had clear, easily recorded, and highly reproducible suture movements (Figure 5); whereas in others with apparently more rigid skulls, spontaneous motions similar to those shown in Figure 3 were unabated and unaffected by the externally applied forces.

Our data indicate that not only do the parietal bones move in reference to one another by forces applied externally to the head, but also they are displaced by intracranial forces. Cerebrospinal fluid pressure increases accompanying induced hypercapnia (Figure 6, top frame) or injected norepinephrine (Figure 6, center frame) are closely correlated with lateral if not rotational parietal bone movement during both onset of effect and recovery from it. Similar correspondence is demonstrated when intracranial pressure is increased by bolus injections of artificial CSF (Figure 6, bottom frame).

The strategy of increasing intracranial volume and pressure by injecting artificial CSF into a cerebral ventricle has important advantages over just having the animal breathe $\mathrm{CO}_{2}$ or elevating intracranial pressure with an injected catecholamine. Because the volume of the injectate and its rate of injection are easily controlled, it is comparatively easy to define an animal's response to an abrupt increase in cranial volume, as shown by data in Figure 7. Also, because the acute effects of a bolus injection of artificial CSF are soon overcome 


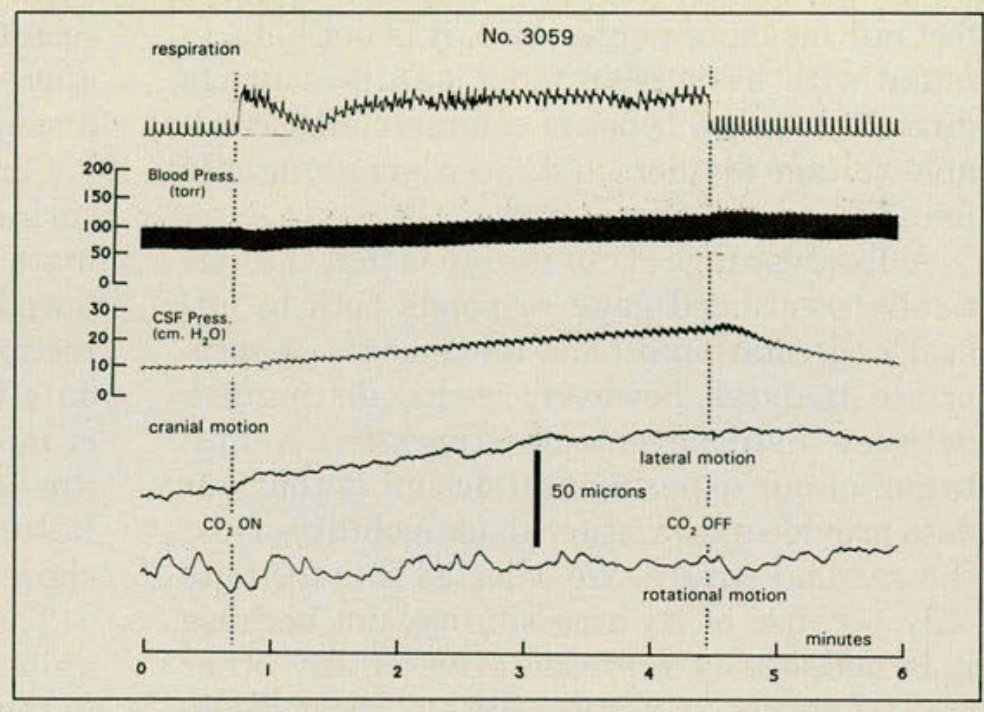

Figure 6. Top frame: Respiration, arterial blood and CSF pressure, and parietal bone movements while an animal breathed an $8 \% \mathrm{CO}_{2}$-inair gas mixture. Calculated for time $=4.4 \mathrm{~min}$ utes, $C M V=64 \mu m$ and $\delta=0.04$ degree. Center frame: Respiratory, cardiovascular, and CSF pressure traces and parietal bone movements while an animal rested spontaneously breathing room air, then received an intravenous $(100 \mu \mathrm{g})$ injection of norepinephrine. Calculated for time $=4.3$ minutes, $C M V=125$ $\mu m$ and $\delta=0.10$ degree. Bottom frame: Respiratory, cardiovascular, and CSF pressure traces and parietal bone movements as the brain's lateral ventricle received a bolus injection of $0.8 \mathrm{~mL}$ of artificial CSF. Calculated at time $=1.0$ minute, $C M V=105 \mu \mathrm{m}$ and $\delta=$ 0.07 degree.

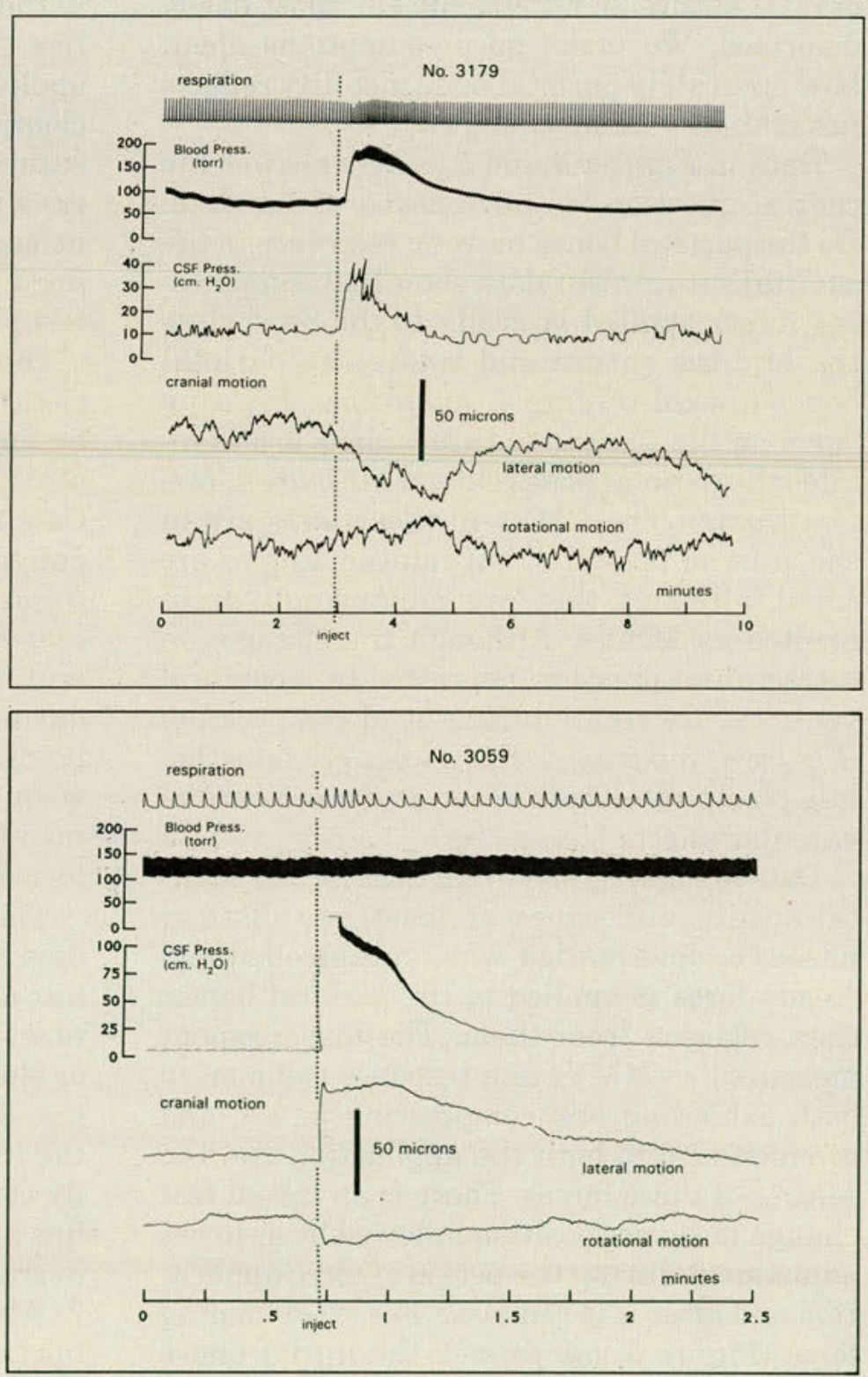




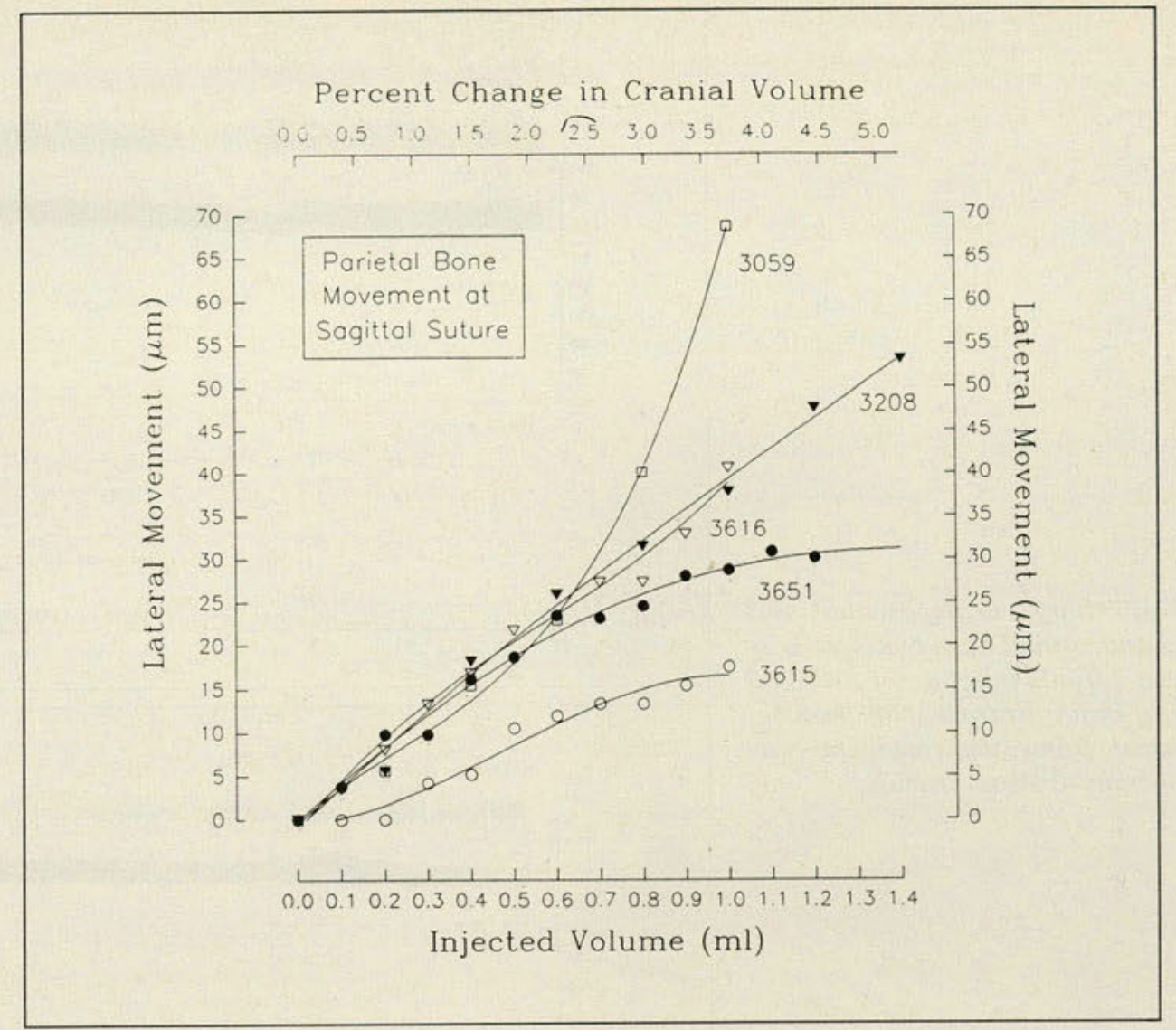

Figure 7. Lateral movement of parietal bones at sagittal suture (ordinate) shown for multiple tests as function of volume of artificial CSF injected into lateral ventricle (bottom abscissa) and percent change in cranial volume it produced (top abscissa). Data are shown for five animals. Lines for each data set were best-fitted by regressional analyses.

(Figure 6, bottom frame), finely graded step-bystep increases in intracranial volume are easy to measure in multiple sequential tests on the same animal. For the experiments that produced data for Figure 7, the effects of each new injected volume were determined only after the animal had returned to a rest level of blood pressure, intracranial pressure, and parietal bone position; all tests were made from the same baseline of control responses.

Data in Figure 7 show that all animals in this test series increased lateral parietal bone movement with successive, controlled increases in intracranial volume. Differences among animals were related to how large a change in MMV there was for any injected volume and to the maximum MMV each animal could sustain. We attribute these differences among animals to factors of cranial compliance.
Our impression is that animals with low compliance (for example, animals No. 3615 and 3651 ; Figure 7 ) showed little additional widening of the sagittal suture once cranial volumes were increased by approximately $4 \%$. Animals with highly compliant skulls (for example, animals No. 3059 and 3208) promised even greater sagittal suture movement had larger volumes of artificial CSF been injected.

We expect that lateral movement of the parietal bones for some animals could be larger than we measured when a volume of artificial $\mathrm{CSF}$ is injected into the cerebral ventricle. The information we have to date (Figure 7) suggests that animals with highly compliant sagittal sutures would allow more suture widening, whereas those with sutures having low compliance have approached the upper limit of suture widening even when cranial volume has increased about $1.0 \mathrm{cc}$. 
Figure 8. Respiratory, cardiovascular, and CSF pressures and cranial bone movements as animal received $1.0-\mathrm{mL}$ injection of artificial CSF into lateral brain ventricle with head secured in stereotaxic frame (top frame) and resting without restraint (bottom frame).
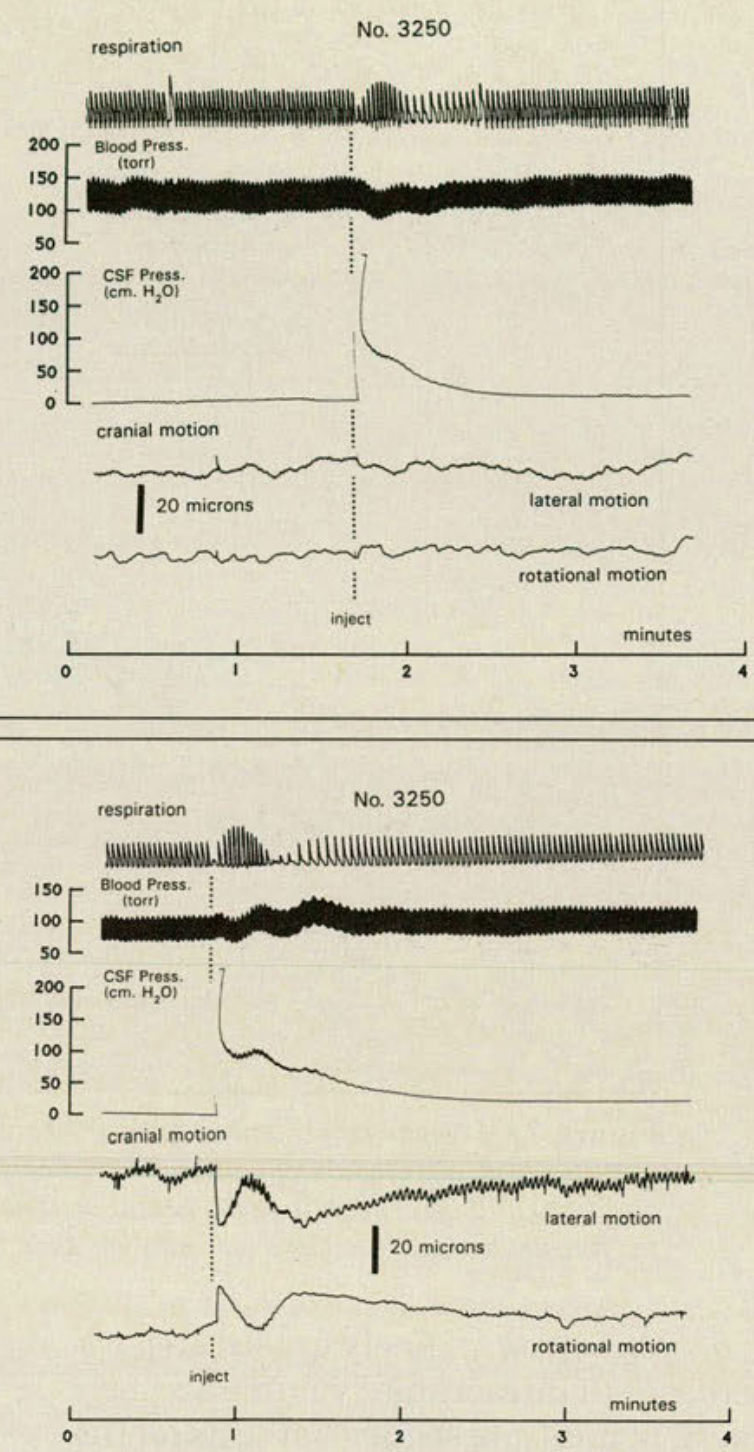

tion to its displacement from the cranium into the spinal subarachnoid space. Cranial suture and total cranial compliances not only are functions of cranial suture mobility, they also depend on the animal's position on its cranial pressure-volume curve.

Cranial bone movement caused by an increase in intracranial pressure depends on more than just the intrinsic compliance of the animal's head, as suggested by data in Figure 8 , top and bottom frames. When artificial CSF was injected intraventricularly, no lateral parietal bone movement or rotation was recorded when the animal's head was restrained (Figure 8 , top frame), but the parietal bones moved 

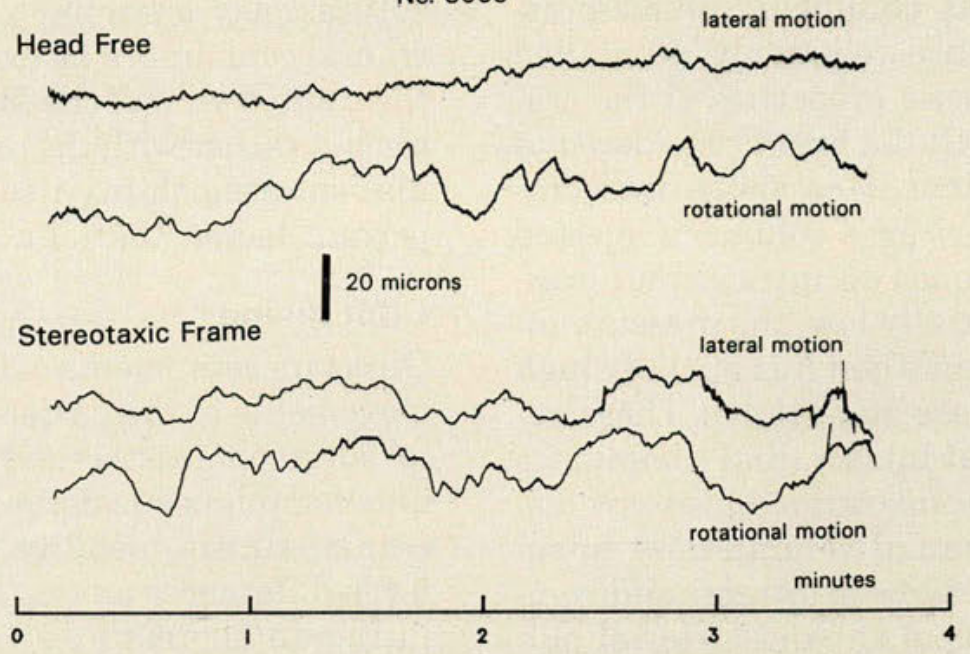

Figure 9. Spontaneous lateral and rotational movements of parietal bones at sagittal suture with head unrestrained (head free) and secured in stereotaxic frame. These sets of data were collected within less than 2 minutes of one another.

laterally and rotationally when the head was released (Figure 8, bottom frame). Respiratory responses were similar in both tests, presumably because the increase in intracranial pressure was the same in both.

Data in Figure 9 also indicate that head restraint is an influential factor in cranial bone motion. Lateral and rotational parietal bone movements were independent of one another when the animal's head was unrestrained. They were more synchronous and MMVs were more similar when cranial motion was restricted by the stereotaxic frame.

Factors that define the hydrodynamic responses of the living cranium are no doubt complex, as are the mechanical properties of the sutures between the skull's bony plates. Brain tissue, the volume of CSF, rates of CSF production and reabsorption, brain blood volume, rates of arterial supply and venous drainage, and the viscoelastic properties of its connective tissue are only a few of the factors that establish statically and dynamically intracranial pressure-volume relationships. ${ }^{22}$

The cranial sutures themselves, however, with their collagen content and degree of in- terdigitation, must play an important role in cranial compliance. Cranial sutures in humans, for example, are more compliant than is cranial bone. ${ }^{23} \mathrm{Also}$, cranial sutures are able to absorb more energy than equivalent volumes of bone ${ }^{24}$ How much a suture resists a bending force depends on the extent to which it is interdigitated, but all bend more than does the cranial bone itself. ${ }^{24}$

A clear impression emerges that cranial sutures not only provide for cranial volume expansion during growth, but are also compliant sites for bending and energy absorption. Data reported here suggest that this compliance not only is a function of lateral movements of adjacent bony plates, but also depends on their rotation at the fulcrum of the suture itself. Cranial bone movements in response to extracranial and intracranial forces may not be as great in the cat as they are in some nonmammalian species, ${ }^{25}$ but, nevertheless, the cat skull is by no means a rigid vault.

Intracranial pressure dynamics are typically studied by experimental biologists from the standpoint that the skull is a rigid container for the brain, its blood volume, and CSF. In- 
jecting controlled volumes of fluid into the intracranial CSF space, however, reveals the system is compliant. Its nonlinear volume-pressure relationships are commonly attributed only to the viscoelastic properties of the cranium's contents, with the brain considered to be the least compliant. How much intracranial pressure changes for a volume of injected fluid, however, depends on intracranial pressure. When it is initially low, the pressure increase is smaller than when it is initially high for the same increase in volume. These responses indicate that intracranial compliance is high when its pressure is low and vice versa. Increases in intracranial volume raise intracranial pressure and lower intracranial compliance. The effect is to enhance arterial pulsations and the effects of respiration in CSF pressure recordings, as shown by data in Figures 6 , bottom frame and 8 , bottom frame.

Despite the incompressibility of intracranial blood and CSF as liquids, their volumes are rapidly displaced and they serve as important spatial and pressure buffers for the cranium. For example, within seconds after intracranial pressure peaks, some CSF leaves the brain by bulk absorption into the venous blood and by displacement into the spinal subarachnoid space. ${ }^{16,17,26}$ Cerebrospinal fluid is produced and its ionic composition is modified by active transport processes at the choroid plexus and elsewhere in the ventricular system. Cerebrospinal volume flow rates driven by these metabolic processes, however, are slow. They are more important in regulating CSF ionic composition than they are in determining its volume. ${ }^{27,28}$ Brain blood volume plays a similar role when its intracranial volume decreases in compensation for an increase in intracranial pressure. The fluid masses of CSF and brain blood volume are important in setting moment-to-moment as well as long-term balances among the tissue masses and fluid volumes that continuously compete for the limited space in the skull. ${ }^{3,16}$

Intracranial blood and CSF volumes are not alone, though, in damping pressure variations related to changes in intracranial volume. Our data show that there is adequate lateral and rotational mobility of the parietal bones at the sagittal suture to contribute to cranial compliance. It is a good guess that other cranial sutures play a similar role. We propose that cranial compliance be examined not just from the limited view of the Monro-Kellie hypothesis, but rather with the consideration that cranial suture mobility itself contributes an important factor, that of a suture compliance.

\section{Conclusion}

Direct measurements of lateral and rotational movements of the parietal bones at the sagittal suture in anesthetized cats suggest that cranial compliance is defined at least in part by cranial suture mobility. Although there are large differences among animals, increases in intracranial pressure and inward lateral compressive forces on the temporal bones have a general effect of causing the parietal bones to change their position relative to one another.

\section{Acknowledgment}

Thanks are expressed to John Behm, DO, and Kelly Ludema, BS, for their valuable help in the early stages of this study; also to the many medical and undergraduate students who generously volunteered technical services throughout the project.

\section{References}

1. Masuda Y, Yohro T: Are there any regularities in cranial sutures? Okajimas Folia Anat Jpn 1987;64:39-46.

2. Bruce DA: The Pathophysiology of Increased Intracranial Pressure. Kalamazoo, Mich, Upjohn 1978.

3. Lofgren J, von Essen C, Zwetnow NN: The pressure-volume curve of the cerebrospinal fluid space in dogs. Acta Neurol Scand 1973;49:557-574.

4. Portnoy HD, Chopp M, Branch C, et al: Cerebrospinal fiuid pulse waveform as an indicator of cerebral autoregulation. $J$ Neurosurg 1982;56:666-678.

5. Upledger JE, Karni Z: Mechano-electric patterns during craniosacral osteopathic diagnosis and treatment. JAOA 1979;78:782791.

6. Retzlaff EW, Michael DK, Roppel RM: Cranial bone mobility. JAOA 1975;74:869-873.

7. Kappler RE: Osteopathy in the cranial field: Its history, scientific basis and current status. The Osteopathic Physician February 1979 , pp 13-18.

8. Frymann VM: Learning difficulties of children viewed in the light of the osteopathic concept. JAOA 1976;76:46-61.

9. Marmarou A, Shulman K, LaMorgese J: Compartmental analysis of compliance and outflow resistance of the cerebrospinal fluid system. $J$ Neurosurg 1975;43:523-534. 
10. Spertell RB: On the transmission of external pressure fluctuations to the cerebrospinal fluid. J Theor Biol 1981;88:309321.

11. Wilkinson HA, Schuman N, Ruggiero J: Nonvolumetric methods of detecting impaired intracranial compliance or reactivity: Pulse width and wave form analysis. $J$ Neurosurg 1979;50:758-767.

12. Adams T, Heisey SR, Smith MC, et al: Thermodynamic technique for the quantification of regional blood flow. Am JPhysiol 1980;238 (Heart Circ Physiol 7): H682-H696.

13. Fencl V, Vale JR, Broch JA: Respiration and cerebral blood flow in metabolic acidosis and alkalosis in humans. $J$ Appl Physiol 1969;27:67-76.

14. Kuhl DE, Reivich M, Alavi A, et al: Local cerebral blood volume determined by three-dimensional reconstruction of radionuclide scan data. Circ Res 1975;36:610-619.

15. Grubb RL, Raichle ME, Eichling JO, et al: The effects of changes in $\mathrm{P}_{\mathrm{a}} \mathrm{CO}_{2}$ on cerebral blood volume, blood flow and vascular transit time. Stroke 1974;5:630-639.

16. Fisher MJ, Heisey SR, Adams T, et al: Cerebrospinal fluid transients induced by hypercapnia. Am J Physiol 245 (Regulatory Integrative Comp Physiol 14):R701-R705, 1983.

17. Heisey SR, Adams T, Fisher MJ, et al: Effect of hypercapnia and cerebral perfusion pressure on cerebrospinal fluid production in cat. Am J Physiol 244 (Regulatory Integrative Comp Physiol 13):R224-R227, 1983.

18. Cutler RWP, Page L, Galicich J, et al: Formation and absorption of cerebrospinal fluid in man. Brain 1968;91:707-720. 19. Marmarou A, Shulman K, Rosende RM: A nonlinear analy- sis of the cerebrospinal fluid system and intracranial pressure dynamics. $J$ Neurosurg 1978;48:332-344.

20. Shapiro K, Fried A, Marmarou A: Biomechanical and hydrodynamic characterization of the hydrocephalic infant. $J$ Neurosurg 1985;63:69-75.

21. Hochwald GM, Epstein F, Malhan C, et al: The role of the skull and dura in experimental feline hydrocephalus. Dev Med Child Neurol 1972;14(Suppl 27):65-69.

22. Lofgren J, Zwetnow NN: Cranial and spinal components of the cerebrospinal fluid pressure-volume curve. Acta Neurol Scand 1973;49:575-585.

23. Hubbard RD: Flexure of layered cranial bone. J Biomech 1971;4:251-263.

24. Jaslow CR: Mechanical properties of cranial sutures. $J$ Biomech 1990;23:313-321.

25. Throckmorton GS, Clarke LK: Intracranial joint movements in the Agamid lizard, Amphibolurus barbata. J Exp Zool 1981;216:25-35.

26. Heisey SR, Held D, Pappenheimer JR: Bulk flow and diffusion in the cerebrospinal fluid system of the goat. Am J Physiol 1962;203:775-781.

27. Heisey SR: Blood-brain barrier: Vertebrates, in Altman PL (ed): Handbook on Respiration and Circulation. Washington, DC, Federation of American Societies for Experimental Biology, 1971, pp 387-390.

28. Heisey SR, Adams T: Cerebrospinal Fluid (HP-41 Program) publication No. 00548C. H-P User's Library. Corvallis, Ore, Hewlett-Packard Co, 1980.

\section{Appendix}

Analysis is required to distinguish lateral from rotational movement of the parietal bones when both the vertical and horizontal gages record motion (that is, when MMV $=\mathrm{CMV}$ ). In reference to the Figure, we determine the amount of lateral suture movement $(D ; \mu \mathrm{m})$ as a function of the simultaneously recorded rotational movement $\left(y_{v s} ; \mu \mathrm{m}\right)$ and lateral movement $\left(x_{h s} ; \mu \mathrm{m}\right)$ measured by our device. In abstract, the lateral motion is:

$$
x_{h s}=D+l_{h} \delta \cos \beta
$$

and the vertical motion is:

$$
y_{v s}=l_{v} \delta \cos \alpha \text {. }
$$

These two equations are solved simultaneously for the lateral suture movement $D$ and the bone rotation angle $\delta$. These are:

and

$$
D=x_{h s}+y_{v s}\left(l_{h} \cos \beta / l_{v} \cos \alpha\right)
$$

$$
\delta=y_{v s} / l_{v} \cos \alpha \text {. }
$$

When $\delta=0$, then, $D=x_{h s}$ and MMV $=$ CMV. When $\delta>0$, then $x_{h s}$ underestimates the lateral displacement of the parietal bones and the widening of the sagittal suture (that is, MMV $<$ CMV). When $\delta<0$, then $x_{h s}$ overestimates lateral parietal bone movement (that is, MMV $>\mathrm{CMV}$ ). The smaller the rotation of the parietal bones at the sagittal suture, the more the voltage output of the vertically positioned strain gage directly reflects their lateral separation, and vice versa. Regardless of how much any inward (negative) or outward (positive) rotational movement affects the recording of lateral bone movement, we calculate their interactive effects.

Our derivations are based on the geometry and nomenclature of Figure 1 (text) and Figure (Appendix). The convention is that $X$ axis for the device is positive and the rotation angle $(\delta)$ is positive counterclockwise. The angles $\alpha$ and $\beta$, and the skull angle $(\gamma)$ are unchanging geometric angles and $\delta$ is the angular movement of the parietal bones around the fulcrum of the sagittal suture where they are joined at the midline dorsal skull surface.

\section{For Sensor 1}

The length from the axis to the stylus point is:

$$
l_{h}=\sqrt{x_{h}{ }^{2}+y_{h}{ }^{2}}
$$

where $x_{h}$ and $y_{h}$ are the distances shown in Figure (Appendix). This length can be used as a radius and because of the small motions, the resulting arc length can be considered to be a straight line. 
Figure. Geometry for analyses made with device for quantifying cranial bone motion. The angles for Sensor $2 \alpha$ and Sensor $1 \beta$ gages along with measured rotational $\left(y_{v}\right)$ and lateral $\left(x_{h}\right)$ movements, the moment arms for rotational $\left(l_{v}\right)$ and lateral $\left(l_{h}\right)$ movements and the angle of rotation $\delta$ and skull contact ( $\gamma$; assumed to be zero) are evaluated in a series of equations to indicate rotational and lateral movement of the cranial bones in reference to the sagittal suture. In the simplest analysis, when rotational movement is zero (as determined by the Sensor 2 strain gages), lateral movement (as determined by Sensor 1 strain gages) defines only horizontal separation of cranial bones in reference to one another. Analyses of related equations deduce how large each of the rotational and lateral movements is when they occur in synchrony.

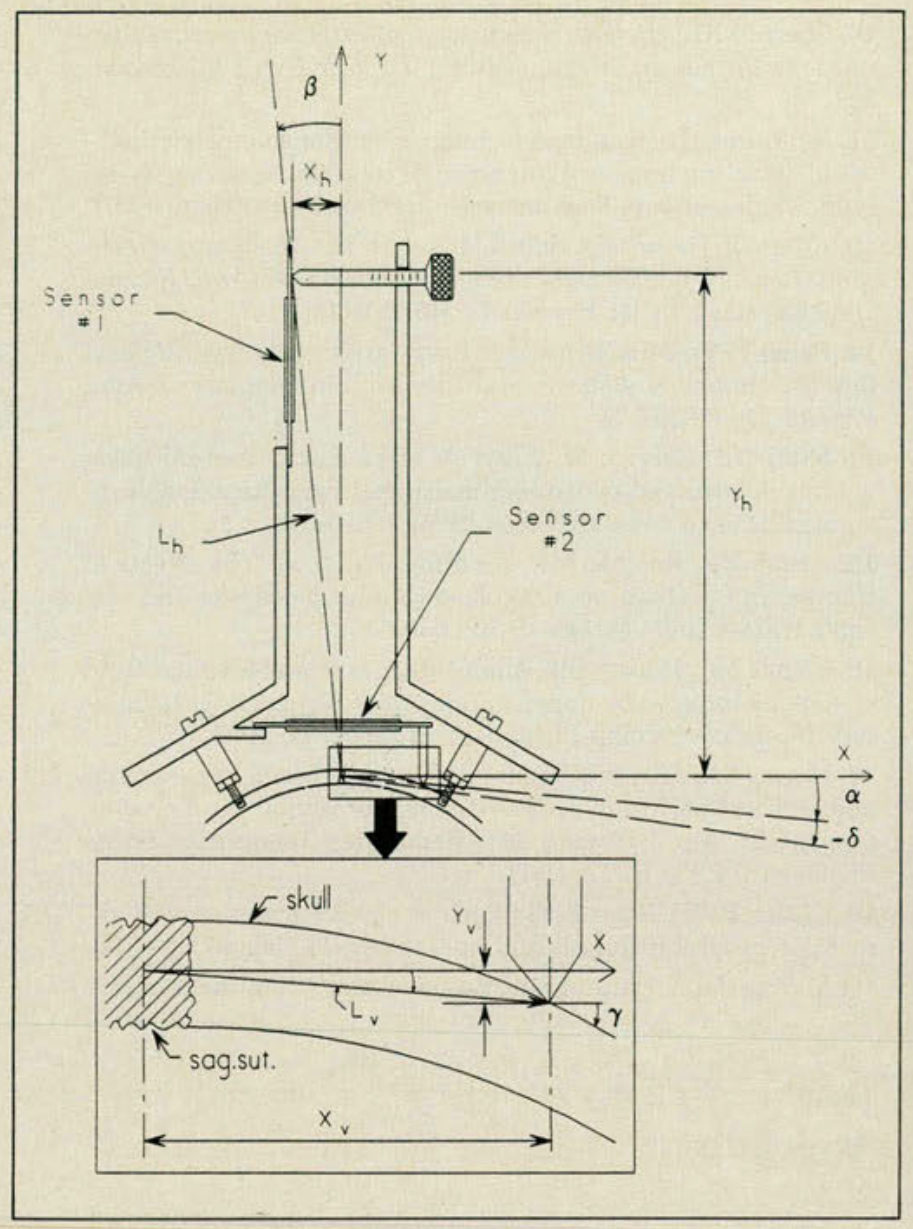

This equation can be solved simultaneously with equation 7 for the unknowns, $D$ and $\delta$.

$$
D=\frac{y_{v s} l_{h} \cos \beta+x_{h s} l_{v} \cos \alpha}{l_{h} \tan \gamma \cos \beta+l_{v} \cos \alpha}
$$

and

$$
\delta=\frac{-x_{h s} \tan \gamma+y_{v s}}{l_{h} \tan \gamma \cos \beta+l_{v} \cos \alpha .}
$$

For this analysis, it is assumed that the horizontal stylus slips on "sensor 1" and the vertical stylus slips on the surface of the parietal bone (Figure 1 [text] and Figure [Appendix]). Equations 3 and 4 were derived on the assumption that skull slope $(\gamma)$ is zero.

The vertical stylus motion due to rotation about the axis of the sagittal suture will be:

$$
y_{v \delta}=\delta l_{v} \cos \alpha \text {. }
$$

The total measured displacement $\left(y_{v s}\right)$ is then:

and if $\gamma=0$, then:

$$
y_{v s}=D \tan \gamma+\delta l_{v} \cos \alpha
$$

$$
y_{v s}=\delta l_{v} \cos \alpha .
$$




\section{cilolz \\ (diltiazem HCl)}
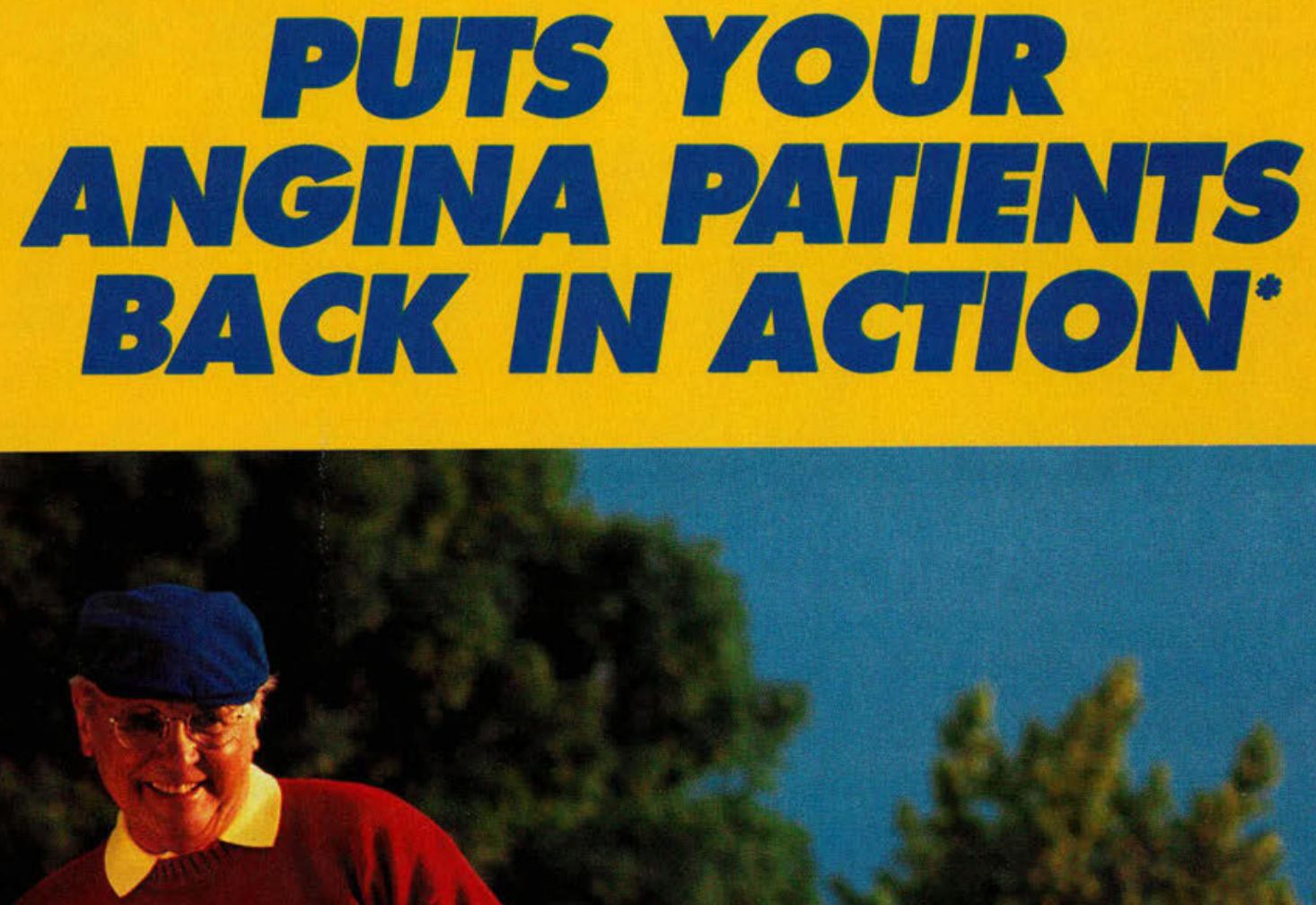

$2.4 \%$

\section{$2.1 \%$

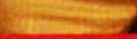

Headache

Edema
.$\%$

\section{$1.5 \%$}

$1.3 \%$

Rash

Asthenia

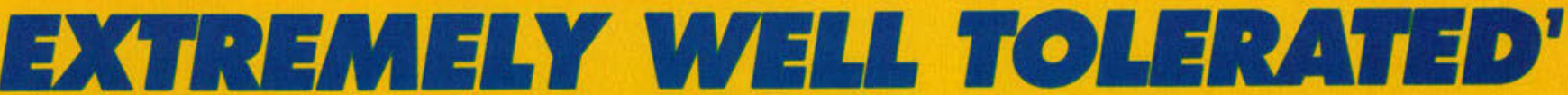

"CARDIZEM" (diltiazem $\mathrm{HCl}$ ) is indicated in the treatment of angina pectoris due to coronary artery spasm and in the management of chronic stable angina (classic effort-associated angina) in patients who cannot tolerate therapy with beta-blockers and/or nitrates or who remain symptomatic despite adequate doses of these agents. 


\section{Unsurpassed reductions in the frequency} of anginal episodes ${ }^{2-5}$ Unsurpassed increases in exercise tolerance $e^{2-4,6}$

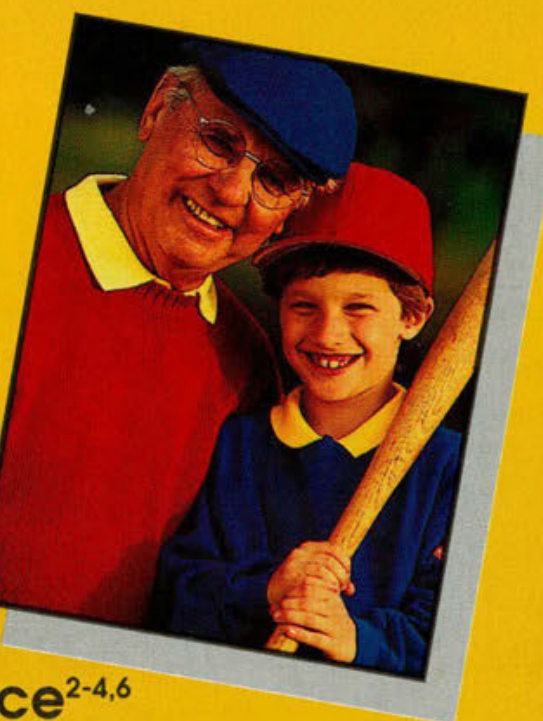

References:

1. Data on file, Marion Merrell Dow Inc. 2. Frishman W. Charlap S, Kimmel B, et al. Circulation. 1988:77:774-786. 3. Klinke WP. Kvill L. Dempsey EE. Grace M. J Am Coll

Cardiol, 1988:12:1562-1567. 4. Hung J, Lamb IH. Connolly SJ. Jutzy KR. Goris ML. Schroeder JS. Circulation. 1983:68:560-567. 5. LAbbate A. Parodi O, Panciroli C, et al.

Cardiology Board Review, 1989:6(suppl):50-54. 6. Anderson JL. Wagner JM, Datz FL. Christian PE, Bray BE, Taylor AT, Am Heart J. 1984:107:698-706.

\section{BRIEF SUMMARY}

\section{CARDIZEM}

(diltiozem hydrochloride) Tablets

\section{CONTRAINDICATIONS}

CARDIZEM is contraindicated in (1) patients with sick sinus syndrome except in the presence of a functioning ventricular pocemaker, (2) patients with second-or third-degree AV block except in the presence of a functioning ventricular pocernaker, (3) patients with hypotension (less than $90 \mathrm{~mm} \mathrm{Hg}$ systolic), (4) patients wh hove demonstrated hypersensitivity to the drug, and (5) patients with acute myocardial infarction and pulmonary congestion documented by $x$-ray on odmission

\section{WARNINGS}

Cordioc Conduction. CARDIZEM prolongs AV node refroctory periods without significantly prolonging sinus node recovery time, except in potients with sick sinus syndrome. This effect may rarely result in abnormally slow heart rates (particularly in patients with sick sinus syndrome) or second- or third-degree AV block (six of 1,243 patients for $0.48 \%$ ). Concomitant use of diticiazern with beto-blockers or digitalis may result in additive effects on cardioc conduction. A patient with Prinzmetal's angina developed periods of asystole ( 2 to 5 seconds) after a single dose of $60 \mathrm{mg}$ of dilitiazem.

2. Congestive Heart Failure. Although ditiazem has a negative inotropic effect in isolated animal tissue preparations, hemody hamic studies in humans with normal ventricular function hove not shown a reduction in cardioc index nor consistent negative effects on contractility $(\mathrm{dp} / \mathrm{dt}$ ) Experience with the use of CARDIZEM alone or in combination with beto-blockers in patients with impoired ventricular function is very limited. Coution should be exercised when using the drug in such patients. Hypotension. Decreoses in blood pressure associated with CARDIZEM theropy may occosionally result in symptomatic hypotension.

4. Acute Hepatic Injury. In rare instances, significant elevations in enzymes such as olkaline phosphatase, IDH, SGOT, SGPT and other phenomena consistent with ocute hepatic injury have been noted. These reactions hove been reversible upon discontinuation of drug therapy. The relationship to CARDIZEM is uncertain in most cases, but probable in some. (See PRECAUTIONS:

\section{PRECAUTIONS}

General. CARDIZEM (dilitizem hydrochioride) is extensively metobolized by the liver and excreted by the kidneys and in bile. As with any drug given over prolonged periods, laboratory parameters should be monitored at regular intervals. The drug should be used with coution in patients with impaired renol or hepatic function. In subocute and chronic dog and rat studies designed to produce toxicity, high doses of diltiazem were associated with hepatic damoge. In special subacute hepatic studies, oral doses of $125 \mathrm{mg} / \mathrm{kg}$ and higher in rats were associated with histological changes in the liver which were reversible when the drug wos discontinued in dogs, doses of $20 \mathrm{mg} / \mathrm{kg}$ were also ossociated with hepatic changes; however, these changes were reversible with continued dosing

Dermatological events (see ADVERSE REACTIONS section) may be transient and may disappear despite continued use of CARDIZEM. However, skin enuptions progressing to erythemo multiforme and/or exfoliative dermotitis have also been intrequently reported. Should a dermatologic reaction persist, the drug should be discontinued.

Drug Interaction. Due to the potential for additive effects, coution and careful titration are worranted in patients receiving CARDIZEM concomitantly with any agents known to offect cordiac controctility and/or conduction. (See WARNINGS )

Pharmacologic studies indicate that there may be odditive effects in prolonging AV conduction when using beta-blockers or digitalis concomitantly with CARDIZEM. (See WARNINGS.

As with all drugs, care should be exercised when treating patients with multiple medications. CARDIZEM undergoes biotransformation by cytochrome P. 450 mixed function oxidose. Coodministration of CARDIZEM with other agents which follow the same route of biotronsformation may result in the competitive inhibition of metabolism. Dosoges of similarly metabolized drugs, particularly those of low therapeutic ratio or in patients with renal and/or hepotic impoirment, may require odjustment when storting or stopping concomitontly odministered CARDIZEM to maintoin optimum theropeutic blood levels

Beta-blockers: Controlled and uncontrolled domestic studies suggest that concomitant use of CARDIZEM and beta-blockers or digitalis is usually well tolercted. Available data ore not sufficient. however, to predict the effects of concomitant treatment, particularly in potients with left ventricular dysfunction or cardioc conduction abnormalities

Administration of CARDIZEM (diltiazem hydrochloride) concomitantly with propranolol in five normal volunteers resulted in increased propranolol levels in all subjects and bioovailability of propranolol wos increosed approximately $50 \%$. If combination theropy is initiated or withdrown in conjunction with propranolol, an adjustment in the propranolol dose may be warranted. (See WARNINGS.

Cimetidine: A study in six heolthy volunteers has shown a significant increase in peak dilitiazem plosma levels $(58 \%)$ and area-under-the-curve ( $53 \%$ ) after o I-week course of cimetidine of $1,200 \mathrm{mg}$ per day and ditiazern $60 \mathrm{mg}$ per day Ranitidine produced smaller, nonsignificant increases. The effect may be me. diated by cimetidine's known inhibition of hepatic cytochrome P-450, the enzyme system probobly responsible for the first-poss metabolism of dittiozem. Patients currently receiving diltiazem therapy should be carefully monitored for a change in phormacological effect when initiating and discontinuing therapy with cimetidine. An adjustment in the diltiazern dose may be warranted

Digitalis: Administration of CARDIZEM with digoxin in 24 healthy male subjects increased plasma digoxin concentrations opproximately $20 \%$. Another investigator found no increose in digoxin levels in 12 patients with coronary artery diseose. Since there have been conflicting results regarding the effect of digoxin levels, it is recommended that digoxin levels be monitored when initioting, adjusting, and discontinuing CARDIZEM therapy to ovoid possible over- or under-digitalization. (See WARNINGS.)

Anesthetics: The depression of cardioc controctility, conductiv. ity and automaficity os well os the voscular dilotion ossociated with anesthetics may be potentiated by calcium channel blockers. When used concomitantly, anesthetics and calcium blockers should be titrated carefully

Corcinogenesis, Mutagenesis, Impaiment of Fertility. A 24. month study in rats and a 21 -month study in mice showed no evidence of carcinogenicity There wos also no mutogenic response in in vitro bacterial tests. No intrinsic effect on fertility wos observed in rats

Pregnancy. Cotegory C. Reproduction studies hove been conducted in mice, rats, and robbits. Administration of doses ranging from five to ten fimes grecter (on a mg/kg bosis) than the daily recommended theropeutic dose hos resulted in embryo and fetol lethality. These doses, in some studies, have been reported to cause skeletal abnormalities. In the perinatal/postnatal studies, there wos some reduction in early individual pup weights and survival rotes. There was on increased incidence of stillbirths of doses of 20 times the human dose or greater.
There are no well-controlled studies in pregnant women: therefore, use CARDIZEM in pregnant women only if the potential benefi: ustifies the potential risk to the fetus.

Nursing Mothers. Diltiazem is excreted in human milk. One report suggests that concentrations in breast milk may approximote serum levels. If use of CARDIZEM is deemed essential, an alternotive method of infant feeding should be instituted.

Pediatric Use. Safety and effectiveness in children have not been established.

\section{ADVERSE REACTIONS}

Serious odverse reactions hove been rare in studies carried out to date, but if should be recognized that patients with impaired ventricular function and cardiac conduction abnormalities have usually been excluded.

In domestic placebo-controlled angina trials, the incidence of odverse reoctions reported during CARDIZEM therapy wos not greater than that reported during placebo theropy.

The following represent occurrences observed in clinicol studies of angina patients. In many coses, the relationship to CARDIZEM hos not been estoblished. The most common occurrences from these studies, os well os their frequency of presentation are: edemo $(2.4 \%)$, headache $(2.1 \%)$, nausea $(1.9 \%)$, dizziness $(1.5 \%)$, rosh (1.3\%), asthenia (1.2\%). In addition, the following events were reported infrequently (less than 1\%)

Cordiovascular: Angina, arrthythmia, AV block (first degree), AV block (second or third degree - see conducfion warning), bradycardia, bundle branch block, congestive heort foilure, ECG abnormalty, flushing, hypotension, palpitations, syncope, tachycardia, ventricular extrosystoles.

Nervous System: Abnormal dreams, amnesia, depression, gait obnormality, hallucinations, insomnia, nervousness, paresthesia, personality change. somnolence, tremor

Gastrointestinal: Anorexia, constipation, diarrheo, dysgeusio. dyspepsia. mild elevations of alkoline phosphotose, SGOT, SGPT, and LDH (see hepatic wornings), thirst, vomiting, weight increose.

Dermatological: Petechioe, photosensitivity, pruritus, urticario

Other: $\quad$ Amblyopio, CPK elevation, dry mouth, dys pnea, epistaxis, eye irritation, hyperglycemio, hyperuricemia, impotence, muscle cromps, nasal congestion, nocturic, osteocrticular pain. polyuria, sexuol dificulties, finnitus.

The following postmarketing events have been reported intrequently in patients receiving CARDIZEM: alopecio, erythema multiforme, extrapyromidal symptoms, gingival hyperplasia, hemolytic anemia, increased bleeding time, leukopenia, purpura, retinopathy, and thrombocylopenia. There have been observed cases of a generalized rosh, charocterized os leukocytoclostic voscultis. In oddition, events such as myocardial inforction have been observed which are not readily distinguishable from the natural history of the diseose in these patients. A definitive cause and effect relationship between these events and CARDIZEM theropy cannot yet be estoblished. Exfoliative dermatitis (proven by rechallenge) hos also been reported.

Issued 1/91

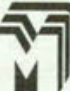

MARION MERRELL DOW INC. PRESCRIPTION PRODUCTS DIVISION 